\title{
Partitions, Durfee Symbols, and the Atkin-Garvan Moments of Ranks
}

\author{
by
}

George E. Andrews *

November 2, 2006

\begin{abstract}
Atkin and Garvan introduced the moments of ranks of partitions in their work connecting ranks and cranks. Here we consider a combinatorial interpretation of these moments. This requires the introduction of a new representation for partitions, the Durfee symbol, and subsequent refinements. This in turn leads us to a variety of new congruences for our 'marked' Durfee symbols much in the spirit of Dyson's original conjectures on the ranks of partitions.
\end{abstract}

\section{Introduction}

In [15], F. J. Dyson, defined the rank of a partition as the largest part minus the number of parts. He conjectured that this partition statistic provided a combinatorial explanation of the famous congruences of Ramanujan. Namely

$$
p(5 n+4) \equiv 0 \quad(\bmod 5)
$$

and

$$
p(7 n+5) \equiv 0 \quad(\bmod 7),
$$

*Partially supported by National Science Foundation Grant DMS 0457003. AMS Classification: 11P83, 05A19, 05A30. Key Words: Partitions, Durfee Symbols, ranks, mock theta functions, moments of ranks 
where $p(n)$ is the number of partitions of $n$.

If we denote the number of partitions of $n$ with rank $m$ by $N(m, n)$ and the number of partitions of $n$ with rank $\equiv m(\bmod Q)$ by $N(m, Q, n)$, then Dyson [15] (cf. [11; pp. 85-87]) showed that

$$
\sum_{n=0}^{\infty} N(m, n) q^{n}=\frac{1}{(q ; q)_{\infty}} \sum_{n=1}^{\infty}(-1)^{n} q^{n(3 n-1) / 2+m n}\left(1-q^{n}\right),
$$

and

$$
\sum_{n=0}^{\infty} N(m, Q, n) q^{n}=\frac{1}{(q ; q)_{\infty}} \sum_{\substack{n=-\infty \\ n \neq 0}}^{\infty}(-1)^{n} q^{n(3 n+1) / 2} \frac{q^{m n}+q^{n(Q-m)}}{1-q^{Q n}}
$$

where

$$
(A ; q)_{\infty}=\prod_{j=0}^{m-1}\left(1-A q^{j}\right)
$$

and

$$
(A ; q)_{\infty}=\lim _{m \rightarrow \infty}(A ; q)_{m}
$$

These results follow easily from the identities:

$$
\begin{aligned}
R_{1}(z ; q) & :=1+\sum_{m=-\infty}^{\infty} \sum_{n=1}^{\infty} N(m, n) z^{m} q^{n} \\
(1.7) & =\sum_{n=0}^{\infty} \frac{q^{n^{2}}}{(z q ; q)_{n}\left(z^{-1} q ; q\right)_{n}} \\
(1.8) & =\frac{1}{(q ; q)_{\infty}}\left(1+\sum_{n=1}^{\infty}(-1)^{n} q^{n(3 n+1) / 2} \frac{\left(1+q^{n}\right)(1-z)\left(1-z^{-1}\right)}{\left(1-z q^{n}\right)\left(1-z^{-1} q^{n}\right)}\right) \\
(1.9) & =\frac{1}{(q ; q)_{\infty}}\left(1+\sum_{n=1}^{\infty}(-1)^{n} q^{n(3 n+1) / 2}\left(\frac{1-z}{1-z q^{n}}+\frac{1-z^{-1}}{1-z^{-1} q^{n}}\right)\right) \\
(1.10) & =\frac{(1-z)}{(q ; q)_{\infty}} \sum_{n=-\infty}^{\infty} \frac{(-1)^{n} q^{n(3 n+1) / 2}}{1-z q^{n}} .
\end{aligned}
$$


The first equality follows by direct combinatorial argument wherein partitions are classified by their Durfee square [20; Ch. 18, §19.7]. The second equality was given by G. N. Watson [30, p. 64], and the last lines follow by partial fractions (cf. Garvan [17; Lemma 7.9]).

Dyson [15] conjectured that for $0 \leqq a \leqq 4, N(a, 5,5 n+4)=p(5 n+4) / 5$ and for $0 \leq a \leqq 6, N(a, 7,7 n+5)=p(7 n+5) / 7$.

Using the above generating functions, Atkin and Swinnerton-Dyer [11] were able to prove all of Dyson's conjectures about the rank.

Dyson's original paper [15] has been a continuing source of inspiration over the years. Partly because of his "crank" conjecture. The rank did not explain Ramanujan's third congruence.

$$
p(11 n+6) \equiv 0 \quad(\bmod 11)
$$

In Dyson's words,

"One is thus led irresistably to the conclusion that there must be some analogue modulo 11 ....

I hold in fact:

That there exists an arithmetical coefficient similar to, but more recondite than, the rank of a partition; I shall call this hypothetical coefficient the "crank" of the partion, and denote by $M(m, q, n)$ the number of partitions of $n$ whose crank is congruent to $m$ modulo $q$ :

that $M(m, q, n)=M(q-m, q, n)$;

that

$$
\begin{gathered}
M(0,11,11 n+6)=M(1,11,11 n+6)=M(2,11,11 n+6) \\
=M(3,11,11 n+6)=M(4,11,11 n+6)
\end{gathered}
$$

that numerous other relations exist analogous to (12)-(19), and in particular

$$
\begin{gathered}
M(1,11,11 n+1)=M(2,11,11 n+1)=M(3,11,11 n+1) \\
=M(4,11,11 n+1)
\end{gathered}
$$

that $M(m, 11, n)$ has a generating function not completely different in form from (24);

that the values of the differences such as $M(0,11, n)-M(4,11, n)$ are always extremely small compared with $p(n)$. 
Whether these guesses are warranted by the evidence, I leave to the reader to decide. Whatever the final verdict of posterity may be, I believe the "crank" is unique among arithmetical functions in having been named before it was discovered. May it be preserved from the ignominious fate of the planet Vulcan!"

The discovery of the vector crank by F. Garvan [17] eventually led to the discovery of the crank [9]. Most recently K. Mahlburg [23] has revealed that the crank explains in a grand and glorious manner the infinite panoply of partition congruences discovered and proved by K. Ono et al. [2], [24], [25].

In a paper relating cranks and ranks [10], Atkin and Garvan found that the moments of ranks and cranks were important in the study of further partition congruences. In particular, they define the $k^{\text {th }}$ moment of the rank by

$$
N_{k}(n)=\sum_{m=-\infty}^{\infty} m^{k} N(m, n) .
$$

In light of the fact that $N(m, n)=N(-m, n)$, we see that the odd order moments are all zero.

It turns out that there is a rich combinatorial and enumerative structure associated with the moments of ranks. Actually what is required is a symmetrized $k^{\text {th }}$ moment function

$$
\eta_{k}(n)=\sum_{m=-\infty}^{\infty}\left(\begin{array}{c}
m+\left\lfloor\frac{k-1}{2}\right\rfloor \\
k
\end{array}\right) N(m, n) .
$$

In Section 2, we shall present the necessary background for consideration of the symmetrized $k^{\text {th }}$ moment function. Section 3 is devoted to the definition of new combinatorial representations of partitions; we shall call these objects Durfee symbols. Subsequently we shall refine these symbols to $k$-marked Durfee symbols. Each of the latter will have $k$ Dyson-like ranks associated with it. When $k=1$, the objects in question reduce to classical partitions, and the new rank is precisely Dyson's original rank, i.e. the largest part minus the number of parts.

The next section of the paper is devoted to congruence theorems for the $\eta_{k}(n)$ which have combinatorial explanations. Special corollaries of our results include

$$
N_{2}(n) \equiv \eta_{2}(n) \equiv 0 \quad(\bmod 5) \quad \text { if } n \equiv \pm 1 \quad(\bmod 5)
$$




$$
\begin{array}{lll}
N_{2}(n) \equiv \eta_{2}(n) \equiv 0 & (\bmod 7) & \text { if } n \equiv 1,5 \quad(\bmod 7) \\
N_{4}(n) \equiv \eta_{4}(n) \equiv 0 & (\bmod 7) & \text { if } n \equiv 1,5 \quad(\bmod 7) .
\end{array}
$$

These congruences may, of course, be deduced easily from the work of Atkin and Swinnerton-Dyer [11]. For example

$$
\begin{aligned}
N_{2}(5 n+4) & =\sum_{m=-\infty}^{\infty} m^{2} N(m, 5 n+4) \\
& \equiv N(1,5,5 n+4)-N(2,5,5 n+4)-N(3,5,5 n+4)+N(4,5,5 n+4) \\
& \equiv 0
\end{aligned}
$$

The others can be proved similarly. Our object in Section 7 will be the provision of a Dyson-like combinatorial explanation of such congruences.

The remainder of the paper considers "odd Durfee symbols". These resemble ordinary Durfee symbols with a modified Durfee square and with odd numbers as entries.

\section{Background}

Here we collect several results that will be relevant in subsequent sections.

Theorem 1. $\eta_{k}(n)=0$ if $k$ is odd.

Proof. Let $k=2 \nu+1$

$$
\begin{aligned}
\eta_{2 \nu+1}(n) & =\sum_{m=-\infty}^{\infty}\left(\begin{array}{c}
m+\nu \\
2 \nu+1
\end{array}\right) N(m, n) \\
& =\sum_{m=-\infty}^{\infty}\left(\begin{array}{c}
-m+\nu \\
2 \nu+1
\end{array}\right) N(-m, n) \\
& =(-1)^{2 \nu+1} \sum_{m=-\infty}^{\infty}\left(\begin{array}{l}
m+\nu \\
2 \nu+1
\end{array}\right) N(m, n)
\end{aligned}
$$

(by the symmetry of binomial coefficients and [15; p. 11, eq. (10) ])

$$
=-\eta_{2 \nu+1}(n) \text {. }
$$




\section{Theorem 2.}

$$
\begin{aligned}
\sum_{n=1}^{\infty} \eta_{2 \nu}(n) q^{n} & =\frac{1}{(q ; q)_{\infty}} \sum_{n=1}^{\infty} \frac{\left(1+q^{n}\right)(-1)^{n-1} q^{n(3 n-1) / 2+\nu n}}{\left(1-q^{n}\right)^{2 \nu}} \\
& =\frac{1}{(q ; q)_{\infty}} \sum_{\substack{n=-\infty \\
n \neq 0}}^{\infty} \frac{(-1)^{n-1} q^{n(3 n-1) / 2+\nu n}}{\left(1-q^{n}\right)^{2 \nu}}
\end{aligned}
$$

Proof. If $\nu>0$, then

$$
\begin{aligned}
& \sum_{n=1}^{\infty} \eta_{2 \nu}(n) q^{n}=\frac{1}{(2 \nu) !}\left(\frac{d^{2 \nu}}{d z^{2 \nu}} z^{\nu-1} R_{1}(z ; q)\right)_{z=1} \\
& =\frac{1}{(2 \nu) !} \sum_{j=0}^{\nu-1}\left(\begin{array}{c}
2 \nu \\
j
\end{array}\right)(\nu-1)(\nu-2) \cdots(\nu-j) R_{1}^{(2 \nu-j)}(1 ; q)
\end{aligned}
$$

(by Leibniz's rule).

Now

$$
R_{1}^{\prime}(z ; q)=\frac{-1}{(q ; q)_{\infty}} \sum_{\substack{n=-\infty \\ n \neq 0}}^{\infty} \frac{(-1)^{n} q^{n(3 n+1) / 2}\left(1-q^{n}\right)}{\left(1-z q^{n}\right)^{2}}
$$

and consequently for $j \geqq 1$

$$
R_{1}^{(j)}(z ; q)=\frac{-j !}{(q ; q)_{\infty}} \sum_{\substack{n=-\infty \\ n \neq 0}}^{\infty} \frac{(-1)^{n} q^{n(3 n-1) / 2+j n}\left(1-q^{n}\right)}{\left(1-z q^{n}\right)^{j+1}}
$$

Therefore

$$
\begin{aligned}
& \sum_{n=1}^{\infty} \eta_{2 \nu}(n) q^{n}= \\
& \frac{1}{(q ; q)_{\infty}} \sum_{j=0}^{\nu-1}\left(\begin{array}{c}
\nu-1 \\
j
\end{array}\right) \sum_{\substack{n=-\infty \\
n \neq 0}}^{\infty} \frac{(-1)^{n-1} q^{n(3 n-1) / 2+(2 \nu-j) n}\left(1-q^{n}\right)}{\left(1-q^{n}\right)^{2 \nu-j+1}} \\
& =\frac{1}{(q ; q)_{\infty}} \sum_{\substack{n=-\infty \\
n \neq 0}}^{\infty} \frac{(-1)^{n-1} q^{n(3 n-1) / 2+2 \nu n}}{\left(1-q^{n}\right)^{2 \nu}}\left(1+\frac{q^{-n}}{\left(1-q^{n}\right)^{-1}}\right)^{\nu-1}
\end{aligned}
$$




$$
\begin{aligned}
& =\frac{1}{(q ; q)_{\infty}} \sum_{\substack{n=-\infty \\
n \neq 0}}^{\infty} \frac{(-1)^{n-1} q^{n(3 n+1) / 2+\nu n}}{\left(1-q^{n}\right)^{2 \nu}} \\
& =\frac{1}{(q ; q)_{\infty}}\left(\sum_{n=1}^{\infty} \frac{(-1)^{n-1} q^{n(3 n+1) / 2+\nu n}}{\left(1-q^{n}\right)^{2 \nu}}+\sum_{n=1}^{\infty} \frac{(-1)^{-n-1} q^{n(3 n-1) / 2-\nu n}}{\left(1-q^{-n}\right)^{2 \nu}}\right) \\
& =\frac{1}{(q ; q)_{\infty}} \sum_{n=1}^{\infty} \frac{(-1)^{n-1} q^{n(3 n-1) / 2+\nu n}\left(1+q^{n}\right)}{\left(1-q^{n}\right)^{2 \nu}} .
\end{aligned}
$$

If $\nu=0$, then by (1.13) $\eta_{0}(n)=p(n)$ for $n>0$, while $\eta_{0}(0)=0$. Hence starting with the right-hand side of (2.2) with $\nu=0$

$$
\begin{aligned}
& \frac{1}{(q ; q)_{\infty}} \sum_{\substack{n=-\infty \\
n \neq 0}}^{\infty}(-1)^{n-1} q^{n(3 n-1) / 2} \\
& =\frac{1}{(q ; q)_{\infty}}\left(-(q ; q)_{\infty}+1\right) \\
& =\frac{1}{(q ; q)_{\infty}}-1 \quad(\text { by }[5 ; \text { p. } 11, \text { Cor. } 1.7 \quad]) \\
& =\sum_{n=1}^{\infty} p(n) q^{n} \\
& =\sum_{n=1}^{\infty} \eta_{0}(n) q^{n},
\end{aligned}
$$

as desired.

Our next theorem treats

$$
\begin{aligned}
& R_{k}\left(x_{1}, x_{2}, \ldots, x_{k} ; q\right) \\
& =\sum_{\substack{m_{1}>0 \\
m_{2}, m_{3}, \ldots, m_{k} \geq 0}} \frac{q^{\left(m_{1}+m_{2}+\cdots+m_{k}\right)^{2}+\left(m_{1}+\cdots+m_{k-1}\right)+\left(m_{1}+\cdots+m_{k-2}\right)+\cdots+m_{1}}}{\left(x_{1} q ; q\right)_{m_{1}}\left(\frac{q}{x_{1}} ; q\right)_{m_{1}}\left(x_{2} q^{m_{1}} ; q\right)_{m_{2}+1}\left(\frac{q^{m_{1}}}{x_{2}} ; q\right)_{m_{2}+1}} \\
& \times \frac{1}{\left(x_{3} q^{m_{1}+m_{2}} ; q\right)_{m_{3}+1}\left(\frac{q^{m_{1}+m_{2}}}{x_{3}} ; q\right)_{m_{3}+1} \cdots\left(x_{k} q^{m_{1}+\cdots+m_{k-1}} ; q\right)_{m_{k+1}}\left(\frac{q^{m_{1}+\cdots+m_{k-1}}}{x_{k}} ; q\right)_{m_{k+1}}} .
\end{aligned}
$$


Theorem 3. For $k \geqq 2$,

$$
\begin{aligned}
& R_{k}\left(x_{1}, x_{2}, \ldots, x_{k} ; q\right) \\
& =\frac{1}{(q ; q)_{\infty}} \sum_{n=1}^{\infty} \frac{(-1)^{n-1}\left(1+q^{n}\right)\left(1-q^{n}\right)^{2} q^{3 n(n-1) / 2+k n}}{\prod_{j=1}^{k}\left(1-x_{j} q^{n}\right)\left(1-\frac{q^{n}}{x_{j}}\right)} .
\end{aligned}
$$

Proof. We recall the $k$-fold generalization of Watson's $q$-analog of Whipple's Theorem [5, p. 199, Th. 4]:

For $k \geqq 1$, a nonnegative integer,

$$
\begin{aligned}
& { }_{2 k+4} \phi_{2 k+3}\left[\begin{array}{c}
a, q \sqrt{a},-q \sqrt{a}, b_{1}, c_{1}, b_{2}, c_{2}, \ldots, b_{k}, c_{k}, q^{-N} ; q, \frac{a^{k} q^{k+N}}{b_{1} q_{k} c_{1} \ldots c_{k}} \\
\sqrt{a},-\sqrt{a}, a q / b_{1}, a q / c_{1}, a q / b_{2}, a q / c_{2}, \ldots, a q / b_{k}, a q / c_{k}, a q^{N+1}
\end{array}\right] \\
& \frac{(a q ; q)_{N}\left(a q / b_{k} c_{k} ; q\right)_{N}}{\left(a q / b_{k} ; q\right)_{N}\left(a q / c_{k} ; q\right)_{N}} \sum_{m_{1}, \ldots, m_{k-1} \geqq 0} \frac{\left(a q / b_{1} c_{1} ; q\right)_{m_{1}}\left(a q / b_{2} c_{2} ; q\right)_{m_{2}} \cdots\left(a q / b_{k-1} c_{k-1} ; q\right)_{m_{k-1}}}{(q ; q)_{m_{1}}(q ; q)_{m_{2}} \cdots(q ; q)_{m_{k-1}}} \\
& \cdot \frac{\left(b_{2} ; q\right)_{m_{1}}\left(c_{2} ; q\right)_{m_{1}}\left(b_{3} ; q\right)_{m_{1}+m_{2}}\left(c_{3} ; q\right)_{m_{1}+m_{2}} \cdots\left(b_{k} ; q\right)_{m_{1}+\cdots+m_{k-1}}}{\left(a q / b_{1} ; q\right)_{m_{1}}\left(a q / c_{1} ; q\right)_{m_{1}}\left(a q / b_{2} ; q\right)_{m_{1}+m_{2}}\left(a q / c_{2} ; q\right)_{m_{1}+m_{2}} \cdots\left(a q / b_{k-1} ; q\right)_{m_{1}+\cdots+m_{k-1}}} \\
& \frac{\left(c_{k} ; q\right)_{m_{1}+\cdots+m_{k-1}}}{\left(a q / c_{k-1} ; q\right)_{m_{1}+\cdots+m_{k-1}}} \cdot \frac{\left(q^{-N} ; q\right)_{m_{1}+m_{2}+\cdots+m_{k-1}}}{\left(b_{k} c_{k} q^{-N} / a ; q\right)_{m_{1}+m_{2}+\cdots+m_{k-1}}} \\
& \frac{(a q)^{m_{k-2}+2 m_{k-3}+\cdots+(k-2) m_{1}} q^{m_{1}+m_{2}+\cdots+m_{k-1}}}{\left(b_{2} c_{2}\right)^{m_{1}}\left(b_{3} c_{3}\right)^{m_{1}+m_{2}} \cdots\left(b_{k-1} c_{k-1}\right)^{m_{1}+m_{2}+\cdots+m_{k-2}}}
\end{aligned}
$$

here (cf. $[19$, p. 4])

$$
{ }_{r_{1}} \phi_{r}\left(\begin{array}{c}
a_{0}, a_{1}, \ldots, a_{r} ; q, z \\
b_{1}, \ldots, b_{r}
\end{array}\right)=\sum_{n=0}^{\infty} \frac{\left(a_{0} ; q\right)_{n}\left(a_{1} ; q\right)_{n} \cdots\left(a_{r} ; q\right)_{n} z^{n}}{(q ; q)_{n}\left(b_{1} ; q\right)_{n} \cdots\left(b_{r} ; q\right)_{n}} .
$$

In (2.4), replace $k$ by $k+1$, for $1 \leqq j \leqq k$ set $b_{j}=c_{j}^{-1}=x_{j}$ set $a=1$ and let $b_{k+1}, c_{k+1}$ and $N \rightarrow \infty$. After simplification, we find that

$$
1+\sum_{n=1}^{\infty} \frac{\prod_{j=1}^{k}\left(1-x_{j}\right)\left(1-x_{j}^{-1}\right)}{\prod_{j=1}^{k}\left(1-q^{n} x_{j}\right)\left(1-\frac{q^{n}}{x_{j}}\right)}(-1)^{n} q^{n(3 n-1) / 2+k n}\left(1+q^{n}\right)
$$




$$
\begin{gathered}
=(q ; q)_{\infty} \sum_{m_{1}, \ldots, m_{k} \geqq 0} \prod_{j=2}^{k}\left(1-x_{j}\right)\left(1-x_{j}^{-1}\right) \\
\times \frac{1}{\left(x_{1} q ; q\right)_{m_{1}}\left(\frac{q}{x_{1}} ; q\right)_{m_{1}}\left(x_{2} q^{m_{1}} ; q\right)_{m_{2}+1}\left(\frac{q^{m_{1}}}{x_{2}} ; q\right)_{m_{2}+1} \cdots} \\
\frac{1}{\left(x_{k} q^{m_{1}+\cdots+m_{k-1}} ; q\right)_{m_{k}+1}\left(\frac{q^{m_{1}+\cdots+m_{k-1}}}{x_{k}} ; q\right)_{m_{k}+1}} \\
\times q^{\left(m_{1}+\cdots+m_{k}\right)^{2}+m_{1}+\left(m_{1}+m_{2}\right)+\left(m_{1}+m_{2}+m_{3}\right)+\cdots+\left(m_{1}+m_{2}+\cdots+m_{k-1}\right)} .
\end{gathered}
$$

Now let us examine the term $m_{1}=0$ on the right-hand side of (2.6). Upon inspection and simplification, we observe that it is precisely what we would obtain if, on the right-hand side, we replaced $k$ by $k-1$ and replaced each $x_{i}$ by $x_{i+1}$. Hence we see that

$$
\left.\begin{array}{c}
1+\sum_{n=1}^{\infty} \frac{\prod_{j=1}^{k}\left(1-x_{j}\right)\left(1-x_{j}^{-1}\right)(-1)^{n} q^{n(3 n-1) / 2+k n}\left(1+q^{n}\right)}{\prod_{j=1}^{n}\left(1-q^{n} x_{j}\right)\left(1-\frac{q^{n}}{x_{j}}\right)} \\
-\left(1+\sum_{n=1}^{\infty} \frac{\prod_{j=2}^{k}\left(1-x_{j}\right)\left(1-x_{j}^{-1}\right)(-1)^{n} q^{n(3 n-1) / 2+(k-1) n}\left(1+q^{n}\right)}{\prod_{j=2}^{n}\left(1-q^{n} x_{j}\right)\left(1-\frac{q^{n}}{x_{j}}\right)}\right. \\
\quad \sum_{m_{1}>0} \prod_{j=2}^{k}\left(1-x_{j}\right)\left(1-x_{j}^{-1}\right)
\end{array}\right)
$$


Now we may simplify the left-hand side of (2.7); indeed, it reduces to

$$
\begin{aligned}
& \sum_{n=1}^{\infty} \frac{\prod_{j=2}^{k}\left(1-x_{j}\right)\left(1-x_{j}^{-1}\right)(-1)^{n} q^{n(3 n-1) / 2+(k-1) n}}{\prod_{j=1}^{k}\left(1-q^{n} x_{j}\right)\left(1-\frac{q^{n}}{x_{j}}\right)}\left(1+q^{n}\right) \\
& \quad \times\left(\left(1-x_{1}\right)\left(1-x_{j}^{-1}\right) q^{n}-\left(1-q^{n} x_{1}\right)\left(1-\frac{q^{n}}{x_{1}}\right)\right) \\
& =\prod_{j=2}^{k}\left(1-x_{j}\right)\left(1-x_{j}^{-1}\right) \\
& \times \sum_{n=1}^{\infty} \frac{(-1)^{n-1}\left(1+q^{n}\right)\left(1-q^{n}\right)^{2} q^{n(3 n-1) / 2+(k-1) n}}{\prod_{j=1}^{k}\left(1-q^{n} x_{j}\right)\left(1-\frac{q^{n}}{x_{j}}\right)}
\end{aligned}
$$

Substituting this last expression for the left-hand side of (2.7) and dividing both sides of the result by $\prod_{j=2}^{k}\left(1-x_{j}\right)\left(1-x_{j}^{-1}\right)(q ; q)_{\infty}$ we obtain the desired result.

Corollary 4. $R_{k}\left(x_{1}, x_{2}, \ldots, x_{k} ; q\right)$ is a symmetric function of $x_{1}, x_{2}, \ldots, x_{k}$.

Proof. Immediate from inspection of Theorem 3.

Corollary 5. For $\nu \geqq 1$,

$$
R_{\nu+1}(1,1, \ldots, 1 ; q)=\sum_{n=1}^{\infty} \eta_{2 \nu}(n) q^{n}
$$

Proof. By Theorem 3,

$$
\begin{aligned}
R_{\nu+1}(1,1, \ldots, 1 ; q) & =\frac{1}{(q ; q)_{\infty}} \sum_{n=1}^{\infty} \frac{(-1)^{n-1}\left(1+q^{n}\right) q^{n(3 n-1) / 2+\nu n}}{\left(1-q^{n}\right)^{2 \nu}} \\
& =\sum_{n=1}^{\infty} \eta_{2 \nu}(n) q^{n}
\end{aligned}
$$

by Theorem 2 . 
Corollary 6. For $k \geqq 2$,

$$
\begin{aligned}
& R_{k}\left(x_{1}, x_{2}, \ldots, x_{k} ; q\right) \\
= & \frac{1}{2(q ; q)_{\infty}} \sum_{n=-\infty}^{\infty} \frac{(-1)^{n-1}\left(1+q^{n}\right)\left(1-q^{n}\right)^{2} q^{3 n(n-1) / 2+k n}}{\prod_{j=1}^{k}\left(1-x_{j} q^{n}\right)\left(1-\frac{q^{n}}{x_{j}}\right)} .
\end{aligned}
$$

Proof. In the expansion given in Theorem 3, we see that the summand would $=0$ if $n=0$ and that it is unaltered if $n$ is replaced by $-n$.

\section{Theorem 7.}

$$
R_{k}\left(x_{1}, \ldots, x_{k} ; q\right)=\sum_{i=1}^{k} \frac{R_{1}\left(x_{i} ; q\right)}{\prod_{\substack{j=1 \\ j \neq i}}^{k}\left(x_{i}-x_{j}\right)\left(1-\frac{1}{x_{i} x_{j}}\right)}
$$

Proof. First we note a partial fraction identity

$$
\begin{aligned}
& \frac{(1+w)(1-w)^{2} w^{k-2}}{\prod_{j=1}^{k}\left(1-x_{j} w\right)\left(1-\frac{w}{x_{j}}\right)} \\
= & \sum_{i=1}^{k} \frac{\left(x_{i}-1\right)}{\prod_{\substack{j=1 \\
j \neq i}}^{k}\left(x_{i}-x_{j}\right)\left(1-\frac{1}{x_{i} x_{j}}\right)}\left(\frac{1}{1-x_{i} w}-\frac{x_{i}^{-1}}{1-\frac{w}{x_{i}}}\right) .
\end{aligned}
$$

Therefore

$$
\begin{aligned}
& R_{k}\left(x_{1}, \ldots, x_{k} ; q\right) \\
= & \frac{1}{2(q ; q)_{\infty}} \sum_{n=-\infty}^{\infty}(-1)^{n-1} q^{n(3 n+1) / 2} \sum_{i=1}^{k} \frac{\left(x_{i}-1\right)}{\prod_{\substack{j=1 \\
j \neq i}}^{k}\left(x_{i}-x_{j}\right)\left(1-\frac{1}{x_{i} x_{j}}\right)} \\
\times & \left(\frac{1}{1-x_{i} q^{n}}-\frac{x_{i}^{-1}}{1-\frac{q^{n}}{x_{i}}}\right) \\
= & \frac{1}{(q ; q)_{\infty}} \sum_{n=-\infty}^{\infty}(-1)^{n-1} q^{n(3 n+1) / 2} \sum_{i=1}^{k} \frac{\left(x_{i}-1\right)}{\left(1-x_{i} q^{n}\right) \prod_{\substack{i=1 \\
i \neq j}}^{k}\left(x_{i}-x_{j}\right)\left(1-\frac{1}{x_{i} x_{j}}\right)}
\end{aligned}
$$




$$
=\sum_{i=1}^{k} \frac{R_{1}\left(x_{i} ; q\right)}{\prod_{\substack{j=1 \\ j \neq i}}^{k}\left(x_{i}-x_{j}\right)\left(1-\frac{1}{x_{i} x_{j}}\right)} .
$$

Corollary 8. $R_{2}\left(z, z^{2} ; q\right)$

$$
=\frac{z^{2}}{(1-z)\left(1-z^{3}\right)}\left(R_{1}\left(z^{2} ; q\right)-R_{1}(z ; q)\right) .
$$

Proof. Take $k=2, x_{1}=z, x_{2}=z^{2}$ in Theorem 7 .

Corollary 9. $R_{3}\left(z, z^{2}, z^{3} ; q\right)$

$$
\begin{aligned}
& =\frac{R_{1}(z ; q)}{\left(z-z^{2}\right)\left(z-z^{3}\right)\left(1-z^{-3}\right)\left(1-z^{-4}\right)} \\
& +\frac{R_{1}\left(z^{2} ; q\right)}{\left(z^{2}-z\right)\left(z^{2}-z^{3}\right)\left(1-z^{-3}\right)\left(1-z^{-5}\right)} \\
& +\frac{R_{1}\left(z^{3} ; q\right)}{\left(z^{3}-z\right)\left(z^{3}-z^{2}\right)\left(1-z^{-4}\right)\left(1-z^{-5}\right)} .
\end{aligned}
$$

Proof. Take $k=3, x_{1}=z, x_{2}=z^{2}$ and $x_{3}=z^{3}$ in Theorem 7 .

\section{The Durfee Symbol}

There are numerous ways of representing a partition of an integer. For example, the partition $5+5+4+4+3+1$ of 22 , has a Ferrers graph $[6, \S 1.3]$

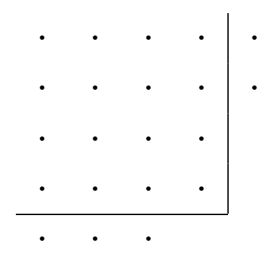

The largest square of nodes in the Ferrers graph is called the Durfee square [6, p. 28]. 
There is also the Frobenius symbol

$$
\left(\begin{array}{llll}
4 & 3 & 1 & 0 \\
5 & 3 & 2 & 0
\end{array}\right)
$$

where the top line indicates the rows to the right of the main diagonal in the Ferrers graph and the bottom line indicates the columns below, viz.

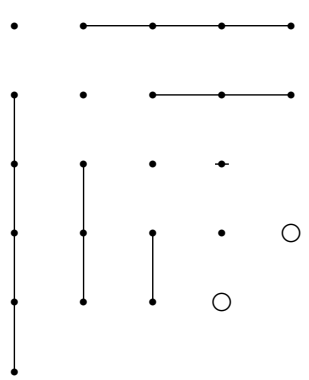

Note that the numbers being partitioned (i.e. 22) is equal to the sum of both rows plus the number of nodes on the main diagonal $($ i.e. $8+10+4)$. We know from Jacobi's identity [6, p. 28]

$$
\begin{aligned}
\sum_{n=0}^{\infty} p(n) q^{n}=\frac{1}{(q ; q)_{\infty}} & =\sum_{n=0}^{\infty} \frac{q^{n^{2}}}{(q ; q)_{n}^{2}} \\
& =\sum_{n=0}^{\infty} q^{n} \cdot \frac{q^{\frac{n(n-1)}{2}}}{(q ; q)_{n}} \cdot \frac{q^{\frac{n(n-1)}{2}}}{(q ; q)_{n}}
\end{aligned}
$$

that the Frobenius symbols are, in fact, naturally generated from the final entry in (3.1). However examination of the penultimate series in (3.1) suggests a new symbol for representing partitions. Namely

$$
\left(\begin{array}{ll}
2 & \\
3 & 1
\end{array}\right)_{4},
$$

where the top row consists of the columns to the right of the Durfee square; the bottom row consists of the rows below the Durfee square 


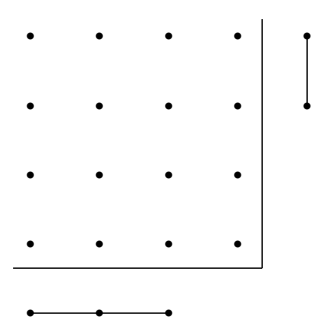

and the subscript 4 denotes the side length of the Durfee square. In this case, the number being partitioned is equal to the sum of the rows of the symbol plus the number of nodes in the Durfee square (i.e. $2+(3+1)+4^{2}=22$ ).

Sylvester's analysis of (3.1) combinatorially introduced the name Durfee square, and consequently we naturally name our new symbol the Durfee symbol.

For example, when $n=5$ 


\begin{tabular}{|c|c|c|c|}
\hline Partitions & Ferrers graph & Frobenius symbol & Durfee symbol \\
\hline 5 & & $\left(\begin{array}{l}4 \\
0\end{array}\right)$ & $\left(\begin{array}{llll}1 & 1 & 1 & 1 \\
& & & \end{array}\right)$ \\
\hline $4+1$ & $\cdots$ & $\left(\begin{array}{l}3 \\
1\end{array}\right)$ & $\left(\begin{array}{lll}1 & 1 & 1 \\
1 & & \end{array}\right)_{1}$ \\
\hline $3+2$ & & $\left(\begin{array}{ll}2 & 0 \\
1 & 0\end{array}\right)$ & $\left(\begin{array}{l}1 \\
\end{array}\right)_{2}$ \\
\hline $3+1+1$ & . & $\left(\begin{array}{l}2 \\
2\end{array}\right)$ & $\left(\begin{array}{ll}1 & 1 \\
1 & 1\end{array}\right)_{1}$ \\
\hline $2+2+1$ & $\begin{array}{l}\cdots \\
\cdots\end{array}$ & $\left(\begin{array}{ll}1 & 0 \\
2 & 0\end{array}\right)$ & $(1)_{2}$ \\
\hline $2+1+1+1$ & . & $\left(\begin{array}{l}1 \\
3\end{array}\right)$ & $\left(\begin{array}{lll}1 & & \\
1 & 1 & 1\end{array}\right)_{1}$ \\
\hline $1+1+1+1+1$ & . & $\left(\begin{array}{l}0 \\
4\end{array}\right)$ & $\left(\begin{array}{llll}1 & 1 & 1 & 1\end{array}\right.$ \\
\hline
\end{tabular}

Note that the rank of a partition is given by the number of entries in the top row of the Durfee symbol minus the number of entries in the bottom row. 


\section{The Definition of Marked Durfee Symbols and Their Ranks}

There have been many partition studies in which several copies of the integers have been used as parts of the partitions, e.g. [1], [3], [4]. To define " $k$ marked Durfee symbols, we shall require $k$ copies of the integers designated by $\left\{1_{1}, 2_{1}, 3_{1}, \ldots\right\},\left\{1_{2}, 2_{2}, 3_{2}, \ldots\right\}, \ldots,\left\{1_{k}, 2_{k}, 3_{k}, \ldots\right\}$. We now form Durfee symbols as in Section 3, but now we use the $k$ copies of the integers for parts in both rows. In addition, we require that: (1) the sequence of parts and the sequence of subscripts in each row be non-increasing; (2) each of the subscripts $1,2, \ldots, k-1$ occur at least once in the top row, and finally (3) if $M_{1}, N_{2}, \ldots, V_{k-2}, W_{k-1}$ are the largest parts with their respective subscripts in the top row, then all parts in the bottom row with subscript 1 lie $[1, M]$, with subscript 2 lie in $[M, N], \ldots$, with subscript $k-1$ lie in $[V, W]$ and with subscript $k$ lie in $[W, S]$ where $S$ is the side of the Durfee square.

As an example, we exhibit the 21 2-marked Durfee symbols associated with the partitions of 5 .

$$
\begin{aligned}
& \begin{array}{lll}
\left(\begin{array}{ll}
1_{1} 1_{1} 1_{1} 1_{1} \\
\left.1_{2} 1_{1} 1_{1} 1_{1}\right)_{1}
\end{array}\right. & \left(\begin{array}{l}
1_{2} 1_{1} 1_{1} \\
1_{2}
\end{array}\right)_{1} & \left(\begin{array}{l}
1_{2} 1_{1} \\
1_{2} 1_{1}
\end{array}\right)_{1} \\
& \left(\begin{array}{l}
1_{2} 1_{2} 1_{1} \\
1_{1}
\end{array}\right) & \left(\begin{array}{l}
1_{1} 1_{1} \\
1_{2} 1_{2}
\end{array}\right)_{1}
\end{array}
\end{aligned}
$$

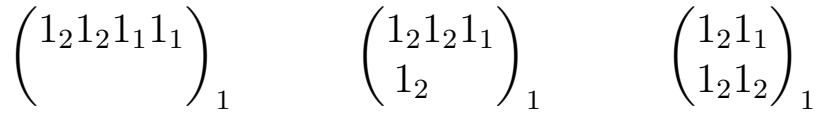

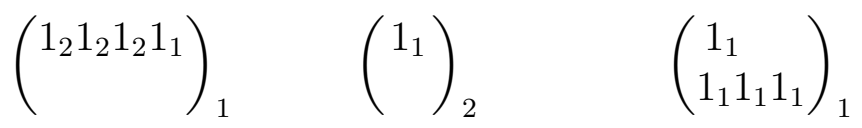

$$
\begin{aligned}
& \left(\begin{array}{l}
1_{1} 1_{1} 1_{1} \\
1_{1}
\end{array}\right)_{1} \quad\left(\begin{array}{l}
1_{1} 1_{1} \\
1_{1} 1_{1}
\end{array}\right)_{1} \quad\left(\begin{array}{l}
1_{1} \\
1_{2} 1_{1} 1_{1}
\end{array}\right)_{1} \\
& \left(\begin{array}{l}
1_{1} 1_{1} 1_{1} \\
1_{2}
\end{array}\right)_{1} \quad\left(\begin{array}{l}
1_{2} 1_{1} \\
1_{1} 1_{1}
\end{array}\right)_{1} \quad\left(\begin{array}{c}
1_{1} \\
1_{2} 1_{2} 1_{1}
\end{array}\right)_{1} \\
& \left(\begin{array}{l}
1_{2} 1_{1} 1_{1} \\
1_{1}
\end{array}\right) \quad\left(\begin{array}{l}
1_{1} 1_{1} \\
1_{2} 1_{1}
\end{array}\right)_{1} \quad\left(\begin{array}{c}
1_{1} \\
1_{2} 1_{2} 1_{2}
\end{array}\right)_{1}
\end{aligned}
$$

Definition. $\mathcal{D}_{k}(n)$ denotes the number of $k$-marked Durfee symbols arising from partitions of $n$. 
In the next section, we shall show that $\mathcal{D}_{k+1}(n)=\eta_{2 k}(n)$. This will form the basis of our subsequent investigations.

To conclude this section we introduce $k$ parameters associated with each $k$-marked Durfee symbol.

Definition. Suppose $\delta$ is a $k$-marked Durfee symbol. We denote the number of entries in the top (resp. bottom) row of $\delta$ by $\tau_{i}(\delta)$ (resp. $\beta_{i}(\delta)$ ). We define $\rho_{i}(\delta)$, the $i$ th $\operatorname{rank}$ of $\delta$, by

$$
\rho_{i}(\delta)= \begin{cases}\tau_{i}(\delta)-\beta_{i}(\delta)-1 & \text { for } 1 \leqq i<k \\ \tau_{i}(\delta)-\beta_{i}(\delta) & \text { for } i=k\end{cases}
$$

Definition. $\mathcal{D}_{k}\left(m_{1}, m_{2}, \ldots, m_{k} ; n\right)$ denotes the number of $k$-marked Durfee symbols arising from partitions of $n$ with $i$ th rank equal to $m_{i}$.

Note that if $k=1$, then $\rho_{1}(\delta)$ is just Dyson's rank and $\mathcal{D}_{1}\left(m_{1} ; n\right)=$ $N(m, n)$.

\section{Generating Functions (1)}

Theorem 10. For $k \geqq 1$

$$
\begin{aligned}
\sum_{n_{1}, n_{2}, \ldots, n_{k}=-\infty}^{\infty} \sum_{n \geq 0} \mathcal{D}_{k}\left(n_{1}, n_{2}, \ldots, n_{k} ; n\right) x_{1}^{n_{1}} x_{2}^{n_{2}} \cdots x_{k}^{n_{k}} q^{n} \\
=R_{k}\left(x_{1}, x_{2}, \ldots, x_{k} ; q\right) .
\end{aligned}
$$

Proof. If $k=1$, this is just (1.7); so in the following we assume $k \geqq 2$. Let us consider a general Durfee symbol wherein the largest entry with subscript 1 on the top row is $m_{1}$, the largest entry on the top row with subscript 2 is $m_{1}+m_{2}, \ldots$, the largest entry on the top row with subscript $k-1$ is $m_{1}+m_{2}+\cdots+m_{k-1}$. Finally the underlying Durfee square has side $m_{1}+m_{2}+\cdots+m_{k}$.

Then parts with subscript 1 on both rows are generated by

$$
\frac{q^{m_{1}}}{\left(x_{1} q ; q\right)_{m_{1}}\left(\frac{q}{x_{1}} ; q\right)_{m_{2}}} .
$$

Parts with subscript 2 on both rows are generated by

$$
\frac{q^{m_{1}+m_{2}}}{\left(x_{2} q^{m_{1}} ; q\right)_{m_{2}+1}\left(\frac{q^{m_{1}}}{x_{2}} ; q\right)_{m_{2}+1}} .
$$


Parts with subscript $k-1$ on both rows are generated by

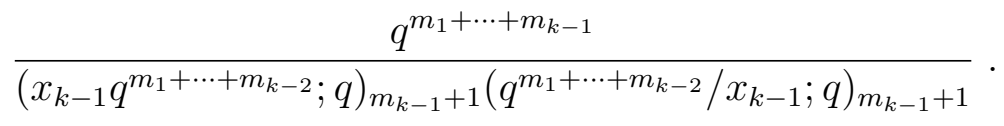

In light of the fact that there are not necessarily any parts with subscript $k$, we see that these parts are generated by

$$
\frac{1}{\left(x_{k} q^{m_{1}+\cdots+m_{k-1}} ; q\right)_{m_{k}+1}\left(q^{m_{1}+\cdots+m_{k-1} / x_{k}} ; q\right)_{m_{k}+1}},
$$

and finally the Durfee square is generated by

$$
q^{\left(m_{1}+m_{2}+\cdots+m_{k}\right)^{2}} .
$$

Multiplying all these expressions together and summing over all $m_{i}$ 's with $m_{1}>0, m_{i} \geqq 0$ for $2 \leqq i \leqq k$, we see that the required generating function is $R_{k}\left(x_{1}, \ldots, x_{k} ; q\right)$ by $(2.3)$.

Corollary 11. For $k \geqq 1$

$$
\begin{aligned}
\sum_{n_{1}, \ldots, n_{k}=-\infty}^{\infty} & \sum_{n=0}^{\infty} \mathcal{D}_{k}\left(n_{1}, n_{2}, \ldots, n_{k} ; n\right) x_{1}^{n_{1}} x_{2}^{n_{2}} \cdots x_{k}^{n_{k}} q^{n} \\
& =\frac{1}{(q ; q)_{\infty}} \sum_{n=1}^{\infty} \frac{(-1)^{n-1}\left(1+q^{n}\right)\left(1-q^{n}\right)^{2} q^{3 n(n-1) / 2+k n}}{\prod_{j=1}^{k}\left(1-x ; q^{n}\right)\left(1-\frac{q^{n}}{x_{j}}\right)}
\end{aligned}
$$

Proof. This follows immediately by combining Theorem 10 with Theorem 3.

Corollary 12. $\mathcal{D}_{k}\left(n_{1}, n_{2}, \ldots, n_{k} ; n\right)$ is symmetric in $n_{1}, n_{2}, \ldots, n_{k}$.

Proof. The generating function as represented in Corollary 11 is clearly symmetric in $x_{1}, x_{2}, \ldots, x_{k}$.

Corollary 13. For $k \geqq 1$,

$$
\mathcal{D}_{k+1}(n)=\eta_{2 k}(n)
$$

Remark. In words, the number of $(k+1)$-marked Durfee symbols related to partitions of $n$ equals the symmetrized $(2 k)$-th moment function at $n$. 
Proof. By Theorem 10 and Theorem 3

$$
\begin{aligned}
\sum_{n \geqq 0} \mathcal{D}_{k+1}(n) q^{n} & =R_{k+1}(1,1, \ldots 1 ; q) \\
& =\sum_{n=1}^{\infty} \eta_{2 k}(n) q^{n}
\end{aligned}
$$

by Corollary 5 .

\section{Self-conjugate $k$-Durfee Symbols}

Sylvester and his student Durfee [27], [28] were the first to study self-conjugate partitions. This led to the analysis of the largest square within the Ferrers graph (named the Durfee square by Sylvester), indeed their analysis of

$$
\sum_{n=0}^{\infty} \frac{q^{n^{2}}}{\left(q^{2} ; q^{2}\right)_{n}}=\left(-q ; q^{2}\right)_{\infty}
$$

led to the now well-known result $[6$, p. 14, Ex. 8] that the number of selfconjugate partitions of $n$ equals the number of partitions of $n$ into distinct odd parts.

Definition. A self-conjugate $k$-Durfee symbol is one in which the two rows are identical, and $S C_{k}(n)$ denotes the number of self-conjugate $k$-Durfee symbols related to $n$. Finally,

$$
\mathcal{S} C_{k}(q)=\sum_{n \geqq 0} S C_{k}(n) q^{n} .
$$

Theorem 14. The number of self-conjugate $k$-Durfee symbols representing $n$ equals the number of partitions of $n$ into distinct unmarked odds and with $(k-1)$ different (albeit possibly repeated) $(k-1)$-marked evens each $\leqq$ twice the number of odd parts.

Proof. Let $\mathcal{O}_{k}(n)$ denote the cardinality of the set of partitions mentioned in the theorem. It is straightforward to provide a combinatorial proof of this result patterned on the Sylvester-Durfee argument [27]. However, to prepare for subsequent results we provide an analytic proof. 
By following exactly the argument in Theorem 10, we see that

$$
\begin{aligned}
& \mathcal{S} C_{k}(q)= \\
& \sum_{\substack{m_{1}>0 \\
m_{2}, \ldots, m_{k} \geqq 0}} \frac{q^{\left(m_{1}+m_{2}+\cdots+m_{k}\right)^{2}+2\left(m_{1}+\cdots+m_{k-1}\right)+2\left(m_{1}+\cdots+m_{k-1}\right)+\cdots+2 m_{1}}}{\left(q^{2} ; q^{2}\right)_{m_{1}}\left(q^{2 m_{1}} ; q^{2}\right)_{m_{2}+1}\left(q^{2\left(m_{1}+m_{2}\right)} ; q^{2}\right)_{m_{3}+1} \cdots\left(q^{2\left(m_{1}+\cdots+m_{k-1}\right)} ; q^{2}\right)_{m_{k}+1}} \\
& =\sum_{M_{k} \geqq 1} \frac{q^{M_{k}^{2}}}{\left(q^{2} ; q^{2}\right)_{M_{k}}} \sum_{1 \leqq M_{1} \leqq M_{2} \leqq \cdots \leqq M_{k}} \frac{q^{2\left(M_{1}+M_{2}+\cdots+M_{k-1}\right)}}{\left(1-q^{2 M_{1}}\right)\left(1-q^{2 M_{2}}\right) \cdots\left(1-q^{\left.2 M_{k-1}\right)}\right.} \\
& =\sum_{n \geqq 0} \mathcal{O}_{k}(n) q^{n},
\end{aligned}
$$

where in the penultimate line we have set $M_{i}-m_{1}+m_{2}+\cdots+m_{i}$, and to obtain the final line we have invoked the Sylvester-Durfee observation that $q^{m^{2}} /\left(q^{2} ; q^{2}\right)_{m}$ is the generating function for partitions with exactly $m$ distinct odd parts.

Theorem 15. For $k \geqq 2$,

$$
\frac{2 \mathcal{S} C_{k}(-q)}{\left(q ; q^{2}\right)_{\infty}}=-\sum_{m=1}^{\infty} \frac{q^{m(m-1) / 2+(k-1)_{m}}\left(1+(-1)^{m}\right)}{(q ; q)_{m}\left(1-q^{m}\right)^{k-1}} .
$$

Proof. To accomplish this result we must invoke Dilcher's wonderful identity $[14$, p. 91, Th. 4]

$$
\begin{aligned}
& \sum_{m=1}^{n} \frac{(-1)^{m-1} q^{m(m+1) / 2+(k-1)_{m}}(q ; q)_{n}}{\left(1-q^{m}\right)^{k}(q ; q)_{m}(q ; q)_{n-m}} \\
= & \sum_{j_{1}=1}^{n} \frac{q^{j_{1}}}{1-q^{j_{1}}} \sum_{j_{2}=1}^{j_{1}} \frac{q^{j_{2}}}{1-q^{j_{2}}} \cdots \sum_{j_{k}=1}^{j_{k-1}} \frac{q^{j_{k}}}{1-q^{j_{k}}}
\end{aligned}
$$

In (6.3), we replace $k$ by $k-1, n$ by $M_{k}$ and $q$ by $q^{2}$. The result is the inner sum in the penultimate line of the proof of Theorem 13.

Hence

$$
\mathcal{S C}_{k}(q)=\sum_{M_{k} \geqq 1} \frac{q^{M_{k}^{2}}}{\left(q^{2} ; q^{2}\right)_{M_{k}}} \sum_{m=1}^{M_{k}} \frac{(-1)^{m-1} q^{m^{2}+m+2(k-2) m}\left(q^{2} ; q^{2}\right)_{M_{k}}}{\left(1-q^{2 m}\right)^{k-1}\left(q^{2} ; q^{2}\right)_{m}\left(q^{2} ; q^{2}\right)_{M_{k}-m}}
$$




$$
\begin{aligned}
& =\sum_{m=1}^{\infty} \frac{(-1)^{m-1} q^{m^{2}+m+2 k m-4 m}}{\left(1-q^{2 m}\right)^{k-1}\left(q^{2} ; q^{2}\right)_{m}} \sum_{M_{k}=0}^{\infty} \frac{q^{\left(M_{k}+m\right)^{2}}}{\left(q^{2} ; q^{2}\right)_{M_{k}}} \\
& =\sum_{m=1}^{\infty} \frac{(-1)^{m-1} q^{2 m^{2}+(2 k-3) m}}{\left(1-q^{2 m}\right)^{k-1}\left(q^{2} ; q^{2}\right)_{m}}\left(-q^{2 m+1} ; q^{2}\right)_{\infty} \\
& =\left(-q ; q^{2}\right)_{\infty} \sum_{m=1}^{\infty} \frac{(-1)^{m-1} q^{2 m^{2}+(2 k-3) m}}{\left(q^{2} ; q^{2}\right)_{m}\left(-q ; q^{2}\right)_{m}\left(1-q^{2 m}\right)^{k-1}} .
\end{aligned}
$$

Therefore

$$
\begin{aligned}
\frac{\mathcal{S} C_{k}(-q)}{\left(q ; q^{2}\right)_{\infty}} & =-\sum_{m=1}^{\infty} \frac{q^{2 m^{2}+(2 k-3) m}}{(q ; q)_{2 m}\left(1-q^{2 m}\right)^{k-1}} \\
& =-\sum_{m=1}^{\infty} \frac{q^{m(m-1) / 2+(k-1) m}}{(q ; q)_{m}\left(1-q^{m}\right)^{k-1}} \frac{1}{2}\left(1+(-1)^{m}\right)
\end{aligned}
$$

and this assertion is clearly equivalent to the desired identity.

\section{Corollary 16.}

$$
\frac{2 \mathcal{S} C_{2}(-q)}{\left(q ; q^{2}\right)_{\infty}}=-\sum_{m=1}^{\infty} \frac{q^{m(m+1) / 2}}{(q ; q)_{m}\left(1-q^{m}\right)}+\sum_{m=1}^{\infty} \frac{q^{m}}{1-q^{m}}
$$

Proof. In light of Theorem 15, we need only show that

$$
\sum_{m=1}^{\infty} \frac{(-1)^{m-1} q^{m(m+1) / 2}}{(q ; q)_{m}\left(1-q^{m}\right)}=\sum_{m=1}^{\infty} \frac{q^{m}}{1-q^{m}}
$$

and this result was proved by both Kluyver [21] and Uchimura [29] (as noted by Dilcher [14]).

In fact, it is known ([7], cf. [8], [14]) that

$$
\sum_{m=1}^{\infty} \frac{(-1)^{m-1} q^{m(m+1) / 2}}{(q ; q)_{m}\left(1-q^{m}\right)^{k-1}}
$$

is representable as an algebraic expression of divisor sums; however, the expression is sufficiently complicated that we have only exhibited the $k=2$ case. 


\section{The Full Rank and Congruences for $\mathcal{D}_{k}(n)$}

In this section, we shall establish the modulus 5 and 7 congruences given in (1.14), (1.15) and (1.16). Indeed we shall prove much more including combinatorial, rank-theoretic explanations of a number of congruences.

Definition. For each $k$-marked Durfee symbol $\delta$, we define the full rank $F R(\delta)$ by

$$
F R(\delta)=\rho_{1}(\delta)+2 \rho_{2}(\delta)+3 \rho_{3}(\delta)+\cdots+k \rho_{k}(\delta),
$$

and we let $N F_{k}(m, n)$ denote the number of $k$-marked Durfee symbols related to $n$ with full rank $m$. In addition, $N F_{k}(m, Q, n)$ denotes the number of $k$ marked Durfee symbols related to $n$ with full rank $\equiv m(\bmod Q)$.

Theorem 17. For $n \geqq 0$ and $a=2,3$ or 5

$N F_{2}(1,5,5 n+a)=N F_{2}(2,5,5 n+a)=N F_{2}(3,5,5 n+a)=N F_{2}(4,5,5 n+a)$,

and for $a=1$ or 4

$$
\begin{array}{r}
N F_{2}(0,5,5 n+a)=N F_{2}(1,5,5 n+a)=N F_{2}(2,5,5 n+a) \\
=N F_{2}(3,5,5 n+a)=N F_{2}(4,5,5 n+a) .
\end{array}
$$

Proof. Clearly by Corollary 8

$$
\sum_{n \geqq 1} \sum_{m=-\infty}^{\infty} N F_{2}(m, n) z^{m} q^{n}=R_{2}\left(z, z^{2} ; q\right)=\frac{1}{\left(z-z^{2}\right)\left(1-z^{-3}\right)}\left(R_{1}(z ; q)-R_{1}\left(z^{2} ; q\right)\right) .
$$

Building on the work of Atkin and Swinnerton-Dyer [11], Garvan [17] established the following formula from Ramaujan't Lost Notebook [26, p. 20]:

$$
\begin{aligned}
R_{1}(\zeta ; q) & =A\left(q^{5}\right)+\left(\zeta+\zeta^{-1}-2\right) \phi\left(q^{5}\right) \\
& +q B\left(q^{5}\right)+\left(\zeta+\zeta^{-1}\right) q^{2} C\left(q^{5}\right) \\
& -\left(\zeta+\zeta^{-1}\right) q^{3}\left(D\left(q^{5}\right)-\left(\zeta^{2}+\zeta^{-2}-2\right) \frac{\psi\left(q^{5}\right)}{q^{5}}\right),
\end{aligned}
$$

where $\zeta$ is any primitive fifth root of unity. Garvan, following Ramanujan, provided and proved explicit beautiful representations for each of $A(q), B(q)$, 
$C(q), D(q), \phi(q)$ and $\psi(q) / q$. For our purposes in this proof we need only know that each of these six functions is an analytic function inside $|q|<1$ with integer coefficients in their power series.

Therefore,

$$
\begin{aligned}
& \sum_{n \geqq 1} \sum_{m=-\infty}^{\infty} N F(m, n) \zeta^{m} q^{n}=R_{2}\left(\zeta, \zeta^{2} ; q\right) \\
& \quad=\frac{1}{\left(\zeta-\zeta^{2}\right)\left(1-\zeta^{-3}\right)}\left(R_{1}(\zeta ; q)-R_{1}\left(\zeta^{2} ; q\right)\right. \\
& \quad=\phi\left(q^{5}\right)+q^{2} C\left(q^{5}\right)-q^{3}\left(D\left(q^{5}\right)-2 \frac{\psi\left(q^{5}\right)}{q^{5}}\right) .
\end{aligned}
$$

Note first that there are no powers of $q$ on the right-hand side that are congruent to either 1 or 4 modulo 5 , and second that if $q$ is real, the righthand side is real.

Collecting terms on the left-hand side that make up the coefficient of $q^{5 n+a}(1 \leqq a \leqq 5)$, we deduce that

$$
\sum_{j=0}^{-1} N F_{2}(j, 5,5 n+a) \zeta^{j}= \begin{cases}0 & \text { if } a=1 \text { or } 4 \\ \text { some real number } & \text { if } a=2,3, \text { or } 5 .\end{cases}
$$

But $\zeta$ is one of the roots of the irreducible polynomial $1+z+z^{2}+z^{3}+z^{4}=$ 0 . Hence the only way the above sum can be true is if the assertions in the Theorem are true; otherwise we would have produced a new irreducible polynomial with $\zeta$ as a root, an impossibility.

There appear to be many more such results. We shall content ourselves with two of the most appealing.

Theorem 18. For $n \geqq 0$ and $0 \leqq i, j \leqq 6$,

$$
N F_{2}(i, 7,7 n+5)=N F_{2}(j, 7,7 n+5),
$$

and

$$
N F_{2}(i, 7,7 n+1)=N F_{2}(j, 7,7 n+1) .
$$

Proof. We note that if $\zeta$ is any primitive seventh root of unity, then

$$
\sum_{j=0}^{6} \zeta^{j} N F_{2}(j, 7,7 n+5)
$$




$$
\begin{aligned}
& =\text { coeff. of } q^{7 n+5} \text { in } R_{2}\left(\zeta, \zeta^{2} ; q\right) \\
& =\text { coeff. of } q^{7 n+5} \text { in } \frac{1}{\left(\zeta-\zeta^{2}\right)\left(1-\zeta^{-3}\right)}\left(R_{1}(\zeta ; q)-R_{1}\left(\zeta^{2} ; q\right)\right) \\
& =\frac{1}{\left(\zeta-\zeta^{2}\right)\left(1-\zeta^{-3}\right)}\left(\sum_{j=0}^{6}\left(\zeta^{j}-\zeta^{2 j}\right) N_{1}(j, 7,7 n+5)\right) \\
& =0
\end{aligned}
$$

where the last line follows from the fact that the $N_{1}(j, 7,7 n+5)$ are identical (by [11, eq. (2.10) and the equation preceeding (2.2)]) and $\zeta$ and $\zeta^{2}$ are both primitive seventh roots of unity. Finally we see that the $N F_{2}(j, 7,7 n+5)$ must all be identical otherwise we would contradict the fact that the minimal polynomial for $\zeta$ is $1+z+z^{2}+z^{3}+z^{4}+z^{5}+z^{6}$.

Next

$$
\begin{aligned}
& \sum_{j=0}^{6} \zeta^{j} N F_{2}(j, 7,7 n+1) \\
& =\text { coeff. of } q^{7 n+1} \text { in } R_{2}\left(\zeta, \zeta^{2} ; q\right) \\
& =\text { coeff. of } q^{7 n+1} \text { in } \frac{1}{\left(\zeta-\zeta^{2}\right)\left(1-\zeta^{-3}\right)}\left(R_{1}(\zeta ; q)-R_{1}\left(\zeta^{2} ; q\right)\right) \\
& =\frac{1}{\left(\zeta-\zeta^{2}\right)\left(1-\zeta^{-3}\right)} \sum_{j=0}^{6}\left(\zeta^{j}-\zeta^{2 j}\right) N_{1}(j, 7,7 n+1) \\
& =\frac{1}{\left(\zeta-\zeta^{2}\right)\left(1-\zeta^{-3}\right)} \sum_{j=1}^{6}\left(\zeta^{j}-\zeta^{2 j}\right) N_{1}(j, 7,7 n+1) \\
& =\frac{N_{1}(1,7,7 n+1)}{\left(\zeta-\zeta^{2}\right)\left(1-\zeta^{-3}\right)} \sum_{j=1}^{6}\left(\zeta^{j}-\zeta^{2 j}\right)
\end{aligned}
$$

(by $[11$, eq. (2.6) and the equation preceeding (2.2)])

$$
\begin{aligned}
& =\frac{N_{1}(1,7,7 n+1)}{\left(\zeta-\zeta^{2}\right)\left(1-\zeta^{-3}\right)} \sum_{j=0}^{6}\left(\zeta^{j}-\zeta^{2 j}\right) \\
& =0
\end{aligned}
$$

and by the same argument used for $N F_{2}(5,7, m+5)$ we see that the $N F_{2}(j, 7,7 n+$ 1) must all be identical. 
Next we provide a similar result for $k=3$.

Theorem 19. For $n \geqq 0,0 \leqq i, j \leqq 6, a=0,1,5$

$$
N F_{3}(i, 7 ; 7 n+a)=N F_{3}(j, 7 ; 7 n+a)
$$

Proof. For simplicity of notation we define $\zeta$ to be any primitive 7 th root of unity, and we let

$$
P(\zeta)=\frac{1}{\zeta^{3}(1-\zeta)\left(1-\zeta^{2}\right)\left(1-\zeta^{3}\right)\left(1-\zeta^{4}\right)} .
$$

If we now take $k=3$, and $z=\zeta$ in ? ? we find that

$$
\begin{aligned}
& R_{3}\left(\zeta, \zeta^{2}, \zeta^{3} ; q\right) \\
& =P(\zeta)\left(\zeta R_{1}(\zeta ; q)-\left(1+\zeta+\zeta^{2}\right) R_{1}\left(\zeta^{2} ; q\right)+\left(1+\zeta^{2}\right) R_{1}\left(\zeta^{3} ; q\right)\right)
\end{aligned}
$$

Consequently

$$
\begin{aligned}
& \sum_{j=0}^{6} \zeta^{j} N F_{3}(j, 7,7 n+a) \\
& =\text { coeff. of } q^{7 n+a} \text { in } R_{3}\left(\zeta, \zeta^{2}, \zeta^{3} ; q\right) \\
& =P(\zeta) \sum_{j=0}^{6}\left(\zeta^{j+1}-\zeta^{2 j}-\zeta^{2 j+1}-\zeta^{2 j+2}+\zeta^{3 j}+\zeta^{3 j+2}\right) N_{1}(j, 7,7 n+a) .
\end{aligned}
$$

Now if $a=5$, then as in Theorem 18, we note that all the $N_{1}(j, 7,7 n+$ $5)$ are identical and immediately the sums all are zero, which leads to the conclusion that the $N_{3}(j, 7,7 n+5)$ are all identical.

If $a=1$, then things are a bit trickier but essentially the same as in the proof of Theorem 18. First we note that

$$
\zeta-1-\zeta-\zeta^{2}+1+\zeta^{2}=0
$$

SO

$$
\sum_{j=0}^{6} \zeta^{j} N F_{3}(j, 7,7 n+1)
$$




$$
\begin{aligned}
& =\text { coeff. of } q^{7 n+1} \text { in } R_{3}\left(\zeta, \zeta^{2}, \zeta^{3} ; q\right) \\
& =P(\zeta) \sum_{j=0}^{6}\left(\zeta^{j+1}-\zeta^{2 j}-\zeta^{2 j+1}-\zeta^{2 j+2}+\zeta^{3 j}+\zeta^{3 j+2}\right) N_{1}(j, 7,7 n+1) \\
& =P(\zeta) \sum_{j=1}^{6}\left(\zeta^{j+1}-\zeta^{2 j}-\zeta^{2 j+1}-\zeta^{2 j+2}+\zeta^{3 j}+\zeta^{3 j+2}\right) N_{1}(j, 7,7 n+1) \\
& =P(\zeta) N_{1}(1,7,7 n+1) \sum_{j=1}^{6}\left(\zeta^{j+1}-\zeta^{2 j}-\zeta^{2 j+1}-\zeta^{2 j+2}+\zeta^{3 j}+\zeta^{3 j+2}\right) \\
& =P(\zeta) N_{1}(1,7,7 n+1) \sum_{j=0}^{6}\left(\zeta^{j+1}-\zeta^{2 j}-\zeta^{2 j+1}-\zeta^{2 j+2}+\zeta^{3 j}+\zeta^{3 j+2}\right) \\
& =0
\end{aligned}
$$

and as before all the $N F_{3}(j, 7,7 n+1)$ are identical.

The most complicated case is $a=0$.

$$
\begin{aligned}
& \sum_{j=0}^{6} \zeta^{j} N_{3}(j, 7,7 n) \\
& =\text { coeff. of } q^{7 n} \text { in } R_{3}\left(\zeta, \zeta^{2}, \zeta^{3} ; q\right) \\
& =P(\zeta) \sum_{j=0}^{6}\left(\zeta^{j+1}-\zeta^{2 j}-\zeta^{2 j+1}-\zeta^{2 j+2}+\zeta^{3 j}+\zeta^{3 j+2}\right) N_{1}(j, 7,7 n) \\
& =P(\zeta)\left(\left(\zeta^{5}-\zeta^{4}\right)\left(N_{1}(1,7,7 n)-N_{1}(6,7,7 n)\right)\right. \\
& \left.\quad+N_{1}(2,7,7 n) \sum_{j=2}^{5}\left(\zeta^{j+1}-\zeta^{2 j}-\zeta^{2 j+1}-\zeta^{2 j+2}+\zeta^{3 j}+\zeta^{3 j+2}\right)\right)
\end{aligned}
$$

(by (7.2) and [11, p. 85, eq. (2.5) and eq's. preceeding (2.2)])

$$
=0
$$

because $N_{1}(1,7,7 n)=N_{1}(6,7,7 n)$ by $[11, \mathrm{p} .85]$ and it is purely a matter of calculation to determine that the remaining $\sum_{j=2}^{5}$ vanishes. Now, as before the third and final assertion of our theorem follows.

Corollary 20. The congurences (1.14), (1.15) and (1.16) are valid. 
Proof. As noted earlier $N_{2}(n)=2 \eta_{2}(n)$. If $n \equiv \pm 1(\bmod 5)$, then by Theorem 17

$$
\eta_{2}(n)=5 N F(0,5 ; n)
$$

if $n \equiv 1$ or $5(\bmod 7)$, then by Theorem 18

$$
\eta_{2}(n)=7 N F(0,7 ; n) .
$$

Finally $N_{4}(n)=24 \eta_{4}(n)+2 \eta_{2}(n)$,

and

$$
\eta_{4}(n)=7 N F_{3}(0,7 ; n)
$$

if $n \equiv 1$ or $5(\bmod 7)$.

\section{$8 \omega(q)$ and Odd Durfee Symbols}

In [30], G. N. Watson defined one of the third order mock theta functions, $\omega(q)$, by

$$
\begin{aligned}
q \omega(q) & :=\sum_{n=0}^{\infty} \frac{q^{2 n(n+1)+1}}{\left(q ; q^{2}\right)_{n+1}^{2}} \\
& =q+2 q^{2}+3 q^{3}+4 q^{4}+6 q^{5}+8 q^{6}+10 q^{7}+14 q^{8}+\cdots .
\end{aligned}
$$

For our purposes, the most convenient partition-theoretic interpretation of $\omega(q)$ is given by considering partitions wherein at least all but one instance of the largest part is one of a pair of consecutive integers. For example, there are 8 such partitions of $6: 6,5+(1+0), 4+(1+0)+(1+0), 3+(2+1)$, $3+(1+0)+(1+0)+(1+0), 2+(2+1)+(1+0), 2+(1+0)+(1+0)+$ $(1+0)+(1+0), 1+(1+0)+(1+0)+(1+0)+(1+0)+(1+0)$.

The fact that $q \omega(q)$ is the generating function for such partitions follows immediately from inspection of Fine's formula for $\omega(q)$ [16, eq. (26.84), p. $61]$

$$
\begin{aligned}
q \omega(q) & =\sum_{n=0}^{\infty} \frac{q^{n+1}}{\left(q ; q^{2}\right)_{n+1}} \\
& =\sum_{n=1}^{\infty} \frac{q^{n}}{\left(1-q^{1+0}\right)\left(1-q^{2+1}\right)\left(1-q^{3+2}\right) \cdots\left(1-q^{n+(n-1)}\right)} .
\end{aligned}
$$


Our study turns out to be most interested in how (8.1) can be seen as naturally generating such partitions. The way to see best what is happening is to invoke P. A. MacMahon's modular partitions [22] with modulus 2 where the dots in the Ferrers graph are replaced by 2's and the graph is bordered by 1's as follows. Let us consider $8+(4+3)+(4+3)+(3+2)+(2+1)+$ $(2+1)+(1+0)+(1+0)+(1+0)$

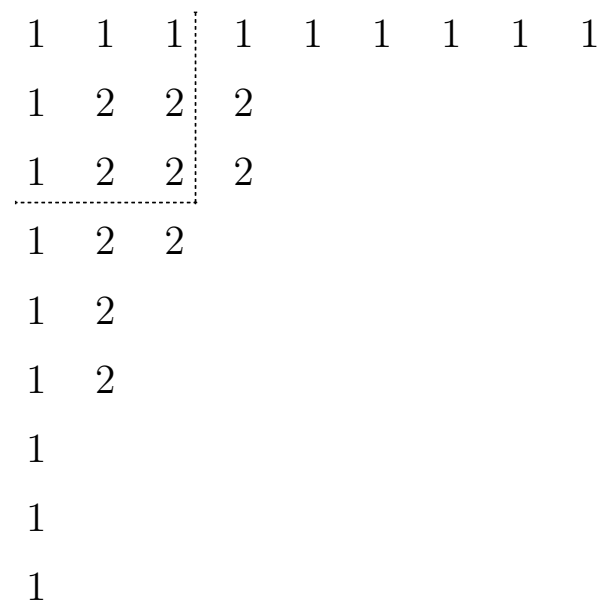

The top row of 1's represents 8; the second row is $(4+3)$ as is the third row; the fourth row is $(3+2)$ followed by two rows each representing $(2+1)$ and the final three singleton rows representing $(1+0)$.

As before, we isolate now the Durfee square of 2's indicated by the dotted lines. In general, a Durfee square of size $n$ will contribute $2 n^{2}+2 n+1$ to the partition (where the $2 n+1$ comes from the bordering 1 's), the rows below the Durfee square yield partitions using consecutive integers the largest of which is $((n+1)+n)$. Equivalently the rows below the Durfee square can be interpreted as partitions with odd parts each $\leqq 2 n+1$. Finally if we read the columns to the right of the Durfee square we also see an arbitrary partition with odd parts each $\leqq 2 n+1$. Consequently the generating function for these modified 2 modular partitions with a Durfee square of side $n$ is given by

$$
\frac{q^{2 n^{2}+2 n+1}}{\left(q ; q^{2}\right)_{n+1}^{2}}
$$

and summing over all $n$ we arrive at (8.1) for the generating function.

Now it becomes quite easy to define a modified Durfee symbol. 
Definition. Each partition into pairs of consecutive integers (excepting one instance of the largest part) has associated with it the odd Durfee symbol

$$
\left(\begin{array}{ll}
a_{1} & a_{2} \cdots a_{i} \\
b_{1} & b_{2} \cdots b_{j}
\end{array}\right)_{n}
$$

wherein the $a$ 's and $b$ 's are all odd numbers each $\leqq 2 n+1$ and the number being partitioned is $2 n^{2}+2 n+1+\sum_{h+1}^{i} a_{h}+\sum_{k=1}^{j} b_{k}$.

Thus in the example we considered originally, the related odd Durfee symbol's

$$
\left(\begin{array}{llllll}
5 & 1 & 1 & 1 & 1 & \\
5 & 3 & 3 & 1 & 1 & 1
\end{array}\right)_{2}
$$

Following Dyson's lead as described in the introduction, we now define the odd rank of an odd Durfee symbol to be the number of entries in the top row minus the number of entries in the bottom row.

In analogy with ordinary Durfee symbols, we let $N^{0}(m, n)$ denote the number of partitions related to an odd Durfee symbol with odd rank $m$, and similar to the ordinary rank

$$
\begin{aligned}
R_{1}^{0}(z ; q) & :=\sum_{n \geqq 1} \sum_{m=-\infty}^{\infty} N^{0}(m, n) z^{m} q^{n} \\
& =\sum_{n \geqq 0} \frac{q^{2 n(n+1)+1}}{\left(z q ; q^{2}\right)_{n+1}\left(z^{-1} q ; q^{2}\right)_{n+1}} .
\end{aligned}
$$

Watson's first identity on page 66 of [30] is equivalent to the following

$$
R_{1}^{0}(z ; q)=\frac{1}{\left(q^{2} ; q^{2}\right)_{\infty}} \sum_{n=-\infty}^{\infty} \frac{(-1)^{n} q^{3 n^{2}+3 n+1}}{1-z q^{2 n+1}} .
$$

We shall not devote a separate section to k-marked odd Durfee symbols because their definition is almost identical to that of the ordinary $k$-marked Durfee symbols. The only change is that: (1) all the entries in the symbol are odd numbers, (2) the subscripting rules are precisely the same, and instead of adding $n^{2}$ to the sum of the entries (as is done for ordinary Durfee symbols) when the Durfee square is of side $n$, we now add $2 n^{2}+2 n+1$ because of the modified Durfee square configuration consisting of an $n \times n$ 
square of 2's bordered by ones.

$$
\begin{array}{ccccc}
1 & 1 & 1 & \cdots & 1 \\
1 & 2 & 2 & \cdots & 2 \\
1 & 2 & 2 & \cdots & 2 \\
\vdots & \vdots & \vdots & & \\
1 & 2 & 2 & \cdots & 2
\end{array}
$$

We shall maintain the conventions of the prior notation and shall add a superscript " 0 " to indicate that we are treating the related object connected with odd $k$-marked Durfee symbols.

\section{Moments of Odd Ranks}

The study of the moments of odd ranks is more straightforward than the results found in Section 2.

Definition. $\eta_{k}^{0}(n)=\sum_{m=-\infty}^{\infty}\left(\begin{array}{c}m+\left\lfloor\frac{k}{2}\right\rfloor \\ k\end{array}\right) N^{0}(m, n)$.

The fact that $\mathcal{O}(m, n)=\mathcal{O}(-m, n)$ (just interchange the rows of the symbol) means that

$$
\eta_{2 m+1}^{0}(n)=0
$$

and the proof is exactly the same as that of Theorem 1.

Theorem 21.

$$
\sum_{n=1}^{\infty} \eta_{2 \nu}^{0}(n) q^{n}=\frac{1}{\left(q^{2} ; q^{2}\right)_{\infty}} \sum_{n=-\infty}^{\infty} \frac{(-1)^{n} q^{3 n^{2}+(2 \nu+3) n+\nu+1}}{\left(1-q^{2 n+1}\right)^{2 \nu+1}}
$$

Proof. Clearly

$$
\begin{aligned}
& \sum_{n=1}^{\infty} \eta_{2 \nu}^{0}(n) q^{n}=\frac{1}{(2 \nu) !}\left(\frac{d^{2 \nu}}{d z^{2 \nu}} z^{\nu} R^{0}(z ; q)\right) \\
& =\frac{1}{(2 \nu) !} \sum_{j=0}^{2 \nu}\left(\begin{array}{c}
2 \nu \\
j
\end{array}\right) \nu(\nu-1) \cdots(\nu-j+1) R^{0^{(2 \nu-j)}}(z ; q)
\end{aligned}
$$

(by Leibniz's rule) 


$$
\begin{aligned}
& =\sum_{j=0}^{\nu}\left(\begin{array}{l}
\nu \\
j
\end{array}\right) \frac{1}{\left(q^{2} ; q^{2}\right)_{\infty}} \sum_{n=-\infty}^{\infty} \frac{(-1)^{n} q^{3 n^{2}+3 n+1}(2 \nu-j)(2 n+1)}{\left(1-q^{2 n+1}\right)^{2 \nu-j+1}} \\
& =\frac{1}{\left(q^{2} ; q^{2}\right)_{\infty}} \sum_{n=-\infty}^{\infty} \frac{(-1)^{n} q^{3 n^{2}+3 n+1+2 \nu(2 n+1)}}{\left(1-q^{2 n+1}\right)^{2 \nu+1}}\left(1+\frac{q^{-(2 n+1)}}{\left(1-q^{2 n+1}\right)^{-1}}\right)^{\nu} \\
& =\frac{1}{\left(q^{2} ; q^{2}\right)_{\infty}} \sum_{n=-\infty}^{\infty} \frac{(-1)^{n} q^{3 n^{2}+(2 \nu+3)+\nu+1}}{\left(1-q^{2 n+1}\right)^{2 \nu+1}}
\end{aligned}
$$

Following the progression of results in Section 2, we define

$$
\begin{aligned}
& \text { (9.1) } \quad R_{k}^{0}\left(x_{1}, x_{2}, \ldots, x_{k} ; q\right) \\
& =\sum_{m_{1}, m_{2}, \ldots, m_{k} \geqq 0} \frac{q^{2\left(m_{1}+m_{2}+\cdots+m_{k}\right)^{2}+2 m_{1}+2\left(m_{1}+m_{2}\right)+\cdots+2\left(m_{1}+m_{2}+\cdots+m_{k}\right)+k}}{\left(x_{1} q ; q^{2}\right)_{m_{1}+1}\left(\frac{q}{x_{1}} ; q^{2}\right)_{m+1}\left(x_{2} q^{2 m_{1}+1} ; q^{2}\right)_{m_{2}+1}\left(\frac{q^{2 m_{1}+1}}{x_{2}} ; q^{2}\right)_{m_{2}+1}} \\
& \frac{1}{\cdots\left(x_{k} q^{2\left(m_{1}+\cdots+m_{k-1}\right)+1} ; q^{2}\right)_{m_{k}+1}\left(\frac{q^{2\left(m_{1}+\cdots+m_{k-1}\right)+1}}{x_{k}} ; q^{2}\right)_{m_{k}+1}}
\end{aligned}
$$

\section{Theorem 22.}

$$
\begin{aligned}
& R_{k}^{0}\left(x_{1}, x_{2}, \ldots, x_{k} ; q\right) \\
& =\frac{1}{\left(q^{2} ; q^{2}\right)_{\infty}} \sum_{n \geqq 0} \frac{\left(1-q^{4 n+2}\right)(-1)^{n} q^{3 n^{2}+(2 k+1) n+k}}{\prod_{i=1}^{k}\left(1-x_{i} q^{2 n+1}\right)\left(1-\frac{q^{2 n+1}}{x_{i}}\right)} .
\end{aligned}
$$

Proof. In (2.4), we replace $q$ by $q^{2}$, then set $a=q^{2}, b_{i}=x_{i} q, c_{i}=q / x_{i}$. Finally we divide both sides by $q^{-k}\left(q^{4} ; q^{2}\right)_{\infty} \prod_{i=1}^{k}\left(1-x_{i} q\right)\left(1-q / x_{i}\right)$ and simplify.

Corollary 23. $R_{k}^{0}\left(x_{1}, \ldots, x_{k} ; q\right)$ is a symmetric function of $x_{1}, x_{2}, \ldots, x_{k}$.

Proof. Immediate from inspection of Theorem 22.

\section{Corollary 24.}

$$
R_{\nu+1}^{0}(1,1, \ldots, 1 ; q)=\sum_{n=1}^{\infty} \eta_{2 \nu}^{0}(n) q^{n}
$$


Proof. By Theorem 22,

$$
\begin{aligned}
R_{\nu+1}^{0}(1,1, \ldots, 1 ; q) & =\frac{1}{\left(q^{2} ; q^{2}\right)_{\infty}} \sum_{n=1}^{\infty} \frac{\left(1+q^{2 n+1}\right)(-1)^{n} q^{3 n^{2}+(2 \nu+3) n+\nu+1}}{\left(1-q^{2 n+1}\right)^{2 \nu+1}} \\
& =\frac{1}{\left(q^{2} ; q^{2}\right)_{\infty}} \sum_{n=-\infty}^{\infty} \frac{(-1)^{n} q^{3 n^{2}+(2 \nu+3) n+\nu+1}}{\left(1-q^{2 n+1}\right)^{2 \nu+1}} \\
& =\sum_{n=1}^{\infty} \eta_{2 \nu}^{0}(n) q^{n}
\end{aligned}
$$

by Theorem 21 .

\section{Theorem 25.}

$$
R_{k}^{0}\left(x_{1}, x_{2}, \ldots, x_{k}\right)=\sum_{i=1}^{k} \frac{R_{1}^{0}\left(x_{i} ; q\right)}{\prod_{\substack{j=1 \\ j \neq 1}}^{k}\left(x_{i}-x_{j}\right)\left(1-\frac{1}{x_{i} x_{j}}\right)}
$$

Proof. First we note that $k=1$ is a tautology. So we now assume $k \geqq 2$. Following the line of proof of Theorem 7, we note a new partial fraction identity

$$
\frac{\left(1-w^{2}\right) w^{k-1}}{\prod_{j=1}^{k}\left(1-x_{j} ; w\right)\left(1-\frac{w}{x_{j}}\right)}=\sum_{i=1}^{k} \frac{1}{\prod_{\substack{j=1 \\ j \neq i}}^{k}\left(x_{i}-x_{j}\right)\left(1-\frac{1}{x_{i} x_{j}}\right)}\left(\frac{1}{1-w x_{i}}+\frac{1}{1-\frac{w}{x_{i}}}\right) .
$$

If we set $w=1$ in this identity, we see that

$$
\sum_{i=1}^{k} \frac{1}{\prod_{\substack{j=1 \\ j \neq 1}}^{k}\left(x_{i}-x_{j}\right)\left(1-\frac{1}{x_{i} x_{j}}\right)}=0
$$

Combining these observations with Theorem 22, we see that

$$
R_{k}^{0}\left(x_{1}, x_{2}, \ldots, x_{k}\right)=\frac{1}{\left(q^{2} ; q^{2}\right)_{\infty}} \sum_{n \geqq 0} \frac{\left(1-q^{4 n+2}\right)(-1)^{n} q^{3 n^{2}+3 n+1+(2 n+1)(k-1)}}{\prod_{j=1}^{k}\left(1-x_{i} q^{2 n+1}\right)\left(1-\frac{q^{2 n+1}}{x_{i}}\right)}
$$




$$
\begin{aligned}
& =\frac{1}{\left(q^{2} ; q^{2}\right)_{\infty}} \sum_{n \geqq 0}(-1)^{n} q^{3 n^{2}+3 n+1} \sum_{i=1}^{k} \frac{1}{\prod_{\substack{j=1 \\
j \neq i}}^{k}\left(x_{i}-x_{j}\right)\left(1-\frac{1}{x_{i} x_{j}}\right)}\left(\frac{1}{1-q^{2 n+1} x_{i}}+\frac{1}{1-\frac{q^{2 n+1}}{x_{i}}}\right) \\
& =\frac{1}{\left(q^{2} ; q^{2}\right)_{\infty}} \sum_{n \geq 0}(-1)^{n} q^{3 n^{2}+3 n+1} \sum_{i=1}^{k} \frac{1}{\prod_{\substack{j=1 \\
j \neq i}}^{k}\left(x_{i}-x_{j}\right)\left(1-\frac{1}{x_{i} x_{j}}\right)} \\
& \times\left(1+\frac{1-q^{4 n+2}}{\left(1-x_{i} q^{2 n+1}\right)\left(1-\frac{q^{2 n+1}}{x_{i}}\right)}\right) \\
& =\frac{1}{\left(q^{2} ; q^{2}\right)_{\infty}} \sum_{n \geqq 0}(-1)^{n} q^{3 n^{2}+3 n+1}\left(1-q^{4 n+2}\right) \sum_{i=1}^{k} \frac{1}{\prod_{\substack{j=1 \\
j \neq i}}^{k}\left(x_{i}-x_{j}\right)\left(1-\frac{1}{x_{i} x_{j}}\right)} \\
& \times \frac{1}{\left(1-x_{i} q^{2 n+1}\right)\left(1-\frac{q^{2 n+1}}{x_{i}}\right)} \\
& \text { (by (9.2) ) } \\
& =\sum_{i=1}^{k} \frac{1}{\prod_{\substack{j=1 \\
j \neq i}}^{k}\left(x_{i}-x_{j}\right)\left(1-\frac{1}{x_{i} x_{j}}\right)} R_{1}^{0}\left(x_{i} ; q\right)
\end{aligned}
$$

\section{Generating Functions (2)}

Theorem 26. For $k \geqq 1$,

$$
\begin{aligned}
& \sum_{n_{1}, n_{2}, \ldots, n_{k}=-\infty} \sum_{n \geqq 0} \mathcal{D}_{k}^{0}\left(n_{1}, n_{2}, \ldots, n_{k}\right) x_{1}^{n} x_{2}^{n_{2}} \cdots x_{k}^{n_{k}} q^{n_{k}} \\
& =R_{k}^{0}\left(x_{1}, x_{2}, \ldots, x_{k} ; q\right) .
\end{aligned}
$$

Proof. The proof here is identical with that of Theorem 10 except for the fact that all parts are odd and the Durfee square given by

$$
\left(m_{1}+m_{2}+\cdots+m_{k}\right)^{2}
$$


is now replaced by the modified square containing

$$
2\left(m_{1}+m_{2}+\cdots+m_{k}\right)^{2}+2\left(m_{1}+m_{2}+\cdots+m_{k}\right)+1
$$

nodes. The rest follows from inspection of (9.1).

Corollary 27. For $k \geqq 1$

$$
\begin{aligned}
& \sum_{n_{1}, \ldots, n_{k}=-\infty} \sum_{n=0}^{\infty} \mathcal{D}_{k}^{0}\left(n_{1}, n_{2}, \ldots, n_{k} ; n\right) x_{1}^{n_{1}} x_{2}^{n_{2}} \cdots x_{k}^{n_{k}} q^{n} \\
= & \frac{1}{\left(q^{2} ; q^{2}\right)_{\infty}} \sum_{n \geqq 0} \frac{\left(1-q^{4 n+2}\right)(-1)^{n} q^{3 n^{2}+(2 k+1) n+k}}{\prod_{i=1}^{k}\left(1-x_{i} q^{2 n+1}\right)\left(1-\frac{q^{2 n+1}}{x_{i}}\right)}
\end{aligned}
$$

Proof. This is immediate by comparing Theorems 26 and 22 .

Corollary 28. $\mathcal{D}_{k}^{0}\left(n_{1}, n_{2}, \ldots, n_{k} ; n\right)$ is symmetric in $n_{1}, n_{2}, \ldots, n_{k}$.

Proof. The generating function as represented in Corollary 27 is clearly symmetric in $x_{1}, x_{2}, \ldots, x_{k}$.

Corollary 29. For $k \geqq 0$,

$$
\mathcal{D}_{k+1}^{0}(n)=\eta_{2 k}^{0}(n)
$$

Remark. In words, the number of $(k+1)$-marked odd Durfee symbols related to $n$ equals the symmetrized $(2 k)$-th moment function at $n$ for odd Durfee symbols.

Proof. By Theorem 26 and Theorem 21

$$
\sum_{n \geqq 0} \mathcal{D}_{k+1}^{0}(n) q^{n}=R_{k+1}^{0}(1,1, \ldots, 1 ; q)=\sum_{n=1}^{\infty} \eta_{2 k}^{0}(n) q^{n}
$$




\section{Self-Conjugate Odd Durfee Symbols}

This section is much shorter than Section 6 owing to the fact that we have been unable to obtain a result for odd summands analogous to Dilcher's formula (6.3).

The definition of self-conjugate $k$-marked Durfee symbols carries over exactly to $k$-marked odd Durfee symbols: the two rows in the symbol are identical.

Furthermore in analogy with (6.2) we see that

$$
\begin{aligned}
& \mathcal{S} C_{k}^{0}(q)= \\
& \sum_{m_{1}, m_{2}, \ldots, m_{k} \geqq 0} \frac{q^{2\left(m_{1}+m_{2}+\cdots+m_{k}\right)^{2}+2\left(m_{1}+\cdots+m_{k}\right)+4\left(m_{1}+\cdots+m_{k-1}\right)+\cdots+4 m_{1}+2 k-1}}{\left(q^{2} ; q^{4}\right)_{m_{1}+1}\left(q^{4 m_{1}+2} ; q^{4}\right)_{m_{2}+1} \cdots\left(q^{4\left(m_{1}+\cdots+m_{k-1}\right)+2} ; q^{4}\right)_{m_{k+1}}} \\
& =\sum_{M_{k} \geqq 0} \frac{q^{2 M_{k}^{2}+2 M_{k}+1}}{\left(q^{2} ; q^{4}\right)_{M_{k+1}}} \sum_{0 \leqq M_{1} \leqq M_{2} \leqq \cdots \leqq M_{k-1}} \frac{q^{4\left(M_{1}+M_{2}+\cdots+M_{k-1}\right)+2(k-1)}}{\left(1-q^{4 M_{1}+2}\right)\left(1-q^{4 M_{2}+2}\right) \cdots\left(1-q^{4 M_{k-1}+2}\right)}
\end{aligned}
$$

It is possible to deduce from (11.1) a theorem analogous to Theorem 13; however, the statement is somewhat complicated. It is of interest to note the following which follows directly from inspection of (11.1).

Lemma 30. $\mathcal{S} C_{k}^{0}(q)$ is an odd function of $q$.

Also one final remark for $k=1$.

$$
\begin{aligned}
\mathcal{S} C_{1}^{0}(q) & =\sum_{m_{1} \geqq 0} \frac{q^{2 m_{1}^{2}+2 m_{1}+1}}{\left(q^{2} ; q^{4}\right)_{m_{1}+1}} \\
& =q v\left(q^{2}\right)
\end{aligned}
$$

where $v(q)$ is another of the third order mock theta functions [30, p. 62].

\section{Parity of Moments of Odd Ranks}

In light of the fact that

$$
R_{1}^{0}(1 ; q)=q \omega(q)
$$


is a mock theta function rather than a modular form, we might expect fewer congruence theorems. In this brief section we shall comment on the parity of the $\mathcal{D}_{k}^{0}(n)$.

We begin by noting a theorem easily deduced from observations of N. J. Fine [16, pp. 61-62]

Theorem 31. $\mathcal{D}_{1}^{0}(n)$ is even except when $n=6 j^{2} \pm 4 j+1$.

Proof. By Theorem 25,

$$
\begin{aligned}
\sum_{n=0}^{\infty} \mathcal{D}_{1}^{0}(n) q^{n} & =R_{1}^{0}(1 ; q) \\
& =q \omega(q) \quad(\text { by }(8.4)) \\
& \left.\equiv q \sum_{n \geqq 0} \frac{(-1)^{n} q^{n}}{\left(q ; q^{2}\right)_{n+1}} \quad(\bmod 2) \quad \text { by }[16, \text { eq. } \quad(26.92)]\right) \\
& =q\left(1+\sum_{j \geqq 1}(-1)^{j} q^{6 j^{2}}\left(q^{4 j}-q^{-4 j}\right)\right) \\
\quad(\text { by }[16, \text { eqs. }) &
\end{aligned}
$$

and the result follows immediately.

We note that for $n<300, \mathcal{D}_{2}(n)$ is odd for $n=2,8,10,16,18,24,34,50,56$, $58,64,66,80,82,88,90,98,104,106,120,128,130,144,154,160,168,170,186,192$, $200,202,216,218,226,234,240,242,248,258,266,274,280,282,288,296$. If parity were distributed randomly we would expect something on the order of 150 odd values instead of 45 .

We note that for $n<300, \mathcal{D}_{3}(n)$ is odd for $n=3,5,7,21,31,39,53,63,67$, $69,71,85,95,101,117,131,133,135,149,181,199,223,231,245,255,263,293$. If parity were distributed randomly we would expect something on the order of 150 odd values instead of 27.

At least part of the discrepancies suggested in these calculations can be explained by the following

Theorem 32. For each $k \geqq 1$, if $n \equiv k-1(\bmod 2)$, then $\mathcal{D}_{k}^{0}(n)$ is even.

Proof. First we note that $\left(\begin{array}{c}2 k \\ 2 j+1\end{array}\right)$ is always even because

$$
(1+x)^{2 k}=\left(1+2 x+x^{2}\right)^{k}
$$




$$
\equiv\left(1+x^{2}\right)^{k} \quad(\bmod 2)
$$

The assertion in our theorem is equivalent to the assertion that

$$
\frac{1}{2}\left(R_{k}^{0}(1, \ldots, 1 ; q)-(-1)^{k} R_{k}^{0}(1, \ldots, 1 ;-q)\right)
$$

has even coefficients, and by Theorem 21

$$
\begin{aligned}
& \frac{1}{2}\left(R_{k}^{0}(1, \ldots, 1 ; q)-(-1)^{k} R_{k}^{0}(1, \ldots, 1 ;-q)\right) \\
& =\frac{1}{2\left(q^{2} ; q^{2}\right)_{\infty}} \sum_{n \geqq 0}\left(1-q^{4 n+2}\right)(-1)^{n} q^{3 n^{2}+(2 k+1) n+k} \\
& \quad \times\left(\frac{1}{\left(1-q^{2 n+1}\right)^{2 k}}-\frac{1}{\left(1+q^{2 n+1}\right)^{2 k}}\right) \\
& =\frac{1}{2\left(q^{2} ; q^{2}\right)_{\infty}} \sum_{n \geqq 0} \frac{(-1)^{n} q^{3 n^{2}+(2 k+1) n+k}}{\left(1-q^{4 n+2}\right)^{2 k-1}} \\
& \quad \times\left(\left(1+q^{2 n+1}\right)^{2 k}-\left(1-q^{2 n+1}\right)^{2 k}\right) \\
& =\frac{1}{\left(q^{2} ; q^{2}\right)_{\infty}} \sum_{n \geqq 0} \frac{(-1)^{n} q^{3 n^{2}+(2 k+1) n+k}}{\left(1-q^{4 n+2}\right)^{2 k-1}} \sum_{j=0}^{k}\left(\begin{array}{c}
2 k \\
2 j+1
\end{array}\right) q^{(2 n+1)(2 j+1)} \\
& \equiv 0 \quad(\bmod 2),
\end{aligned}
$$

which proves our theorem.

Seemingly more is true than is contained in this result.

Conjecture A. $\quad \mathcal{D}_{2}(n)$ is even if $n \equiv 4$ or $6(\bmod 8)$.

Conjecture B. $\quad \mathcal{D}_{3}(n)$ is even if $n \equiv 1,9,11$ or $13(\bmod 16)$.

\section{Mock Theta Functions and Multiple Se- ries}

In light of Theorems 7 and 25, we may find numerous multiple series equal to linear combinations of Ramanujan's mock theta functions. We shall re-

strict our considerations to a few typical examples. Recall the assertions of 
Ramanujan and Watson [30, p. 62]:

$$
\begin{aligned}
f(q) & :=\sum_{n=0}^{\infty} \frac{q^{n^{2}}}{(-q ; q)_{n}^{2}}=R_{1}(-1 ; q) \\
\phi(q) & :=\sum_{n=0}^{\infty} \frac{q^{n^{2}}}{\left(-q^{2} ; q^{2}\right)_{n}}=R_{1}(i ; q) \\
q \omega(q) & :=\sum_{n=0}^{\infty} \frac{q^{2 n^{2}+2 n+1}}{\left(q ; q^{2}\right)_{n+1}^{2}}=R_{1}^{0}(1 ; q) \\
q v\left(q^{2}\right) & :=\sum_{n=0}^{\infty} \frac{q^{2 n^{2}+2 n+1}}{\left(-q^{2} ; q^{4}\right)_{n+1}}=R_{1}^{0}(i ; q) ;
\end{aligned}
$$

also there is the result of Jacobi immediately deduced from (1.7) and (1.8)

$$
\frac{1}{(q ; q)_{\infty}}=R_{1}(1 ; q) .
$$

Hence by Theorem 7 with $k=2$,

$$
\begin{aligned}
& R_{2}(-1,1 ; q)=\frac{1}{4}\left(\frac{1}{(q ; q)_{\infty}}-f(q)\right) \\
& R_{2}(i,-1 ; q)=\frac{1}{2}(\phi(q)-f(q) .
\end{aligned}
$$

By Theorem 25, with $k=2$,

$$
\begin{aligned}
& R_{2}^{0}(-1,1 ; q)=\frac{1}{4}(q \omega(q)-q \omega(-q)) \\
& R_{2}^{0}(i,-1 ; q)=\frac{1}{2}\left(q v\left(q^{2}\right)-q \omega(-q) .\right.
\end{aligned}
$$

By Theorem 7, with $k=3$,

$$
R_{3}(1,-1, i ; q)=\frac{1}{8}\left(\frac{1}{(q ; q)_{\infty}}+f(q)-2 \phi(q)\right),
$$

and by Theorem 25 , with $k=3$

$$
R_{3}^{0}(1,-1, i ; q)=\frac{1}{8}\left(q \omega(q)+q \omega(-q)-q v\left(q^{2}\right)\right) .
$$


While we have written these results succinctly, we remark that in (13.6)(13.9) the left-hand sides are all double series, and in (13.10) and (13.11) the left-hand sides are triple series. For emphasis we exhibit (13.10)

$$
\begin{aligned}
& \sum_{\substack{m_{1}>0 \\
m_{2}, m_{3} \geqq 0}} \frac{q^{\left(m_{1}+m_{2}+m_{3}\right)^{2}+2 m_{1}+m_{2}}}{(q ; q)_{m_{1}}^{2}\left(-q^{m_{1}} ; q\right)_{m_{2}+1}^{2}\left(-q^{2\left(m_{1}+m_{2}\right)} ; q^{2}\right)_{m_{3}+1}} \\
= & \frac{1}{8(q ; q)_{\infty}}+\frac{1}{8} \sum_{n=0}^{\infty} \frac{q^{n^{2}}}{(-q ; q)_{n}^{2}}-\frac{1}{4} \sum_{n=0}^{\infty} \frac{q^{n^{2}}}{\left(-q^{2} ; q^{2}\right)_{n}} .
\end{aligned}
$$

\section{Open Problems}

There exist a variety of serious questions that naturally arise from this study. They fall into 3 basic groups: Combinatorial, asymptotic and congruential.

1. Prove Theorem 7 combinatorially.

2. Prove Corollary 11 combinatorially.

3. Prove Corollary 12 combinatorially.

4. Prove Corollary 13 combinatorially.

5. Prove Theorem 15 combinatorially.

6. Prove Theorem 25 combinatorially.

7. Prove Corollary 27 combinatorially.

8. Prove Corollary 28 combinatorially.

9. Prove Corollary 29 combinatorially.

10. Find a simpler representation for $\mathcal{S} C^{0}(q)$ analogous to the one given for $\mathcal{S C}(q)$ in Theorem 15 .

11. In light of the recent breakthroughs by Bringmann and Ono on the asymptotics for the coefficients in the power series of mock theta functions, [12], provide similar expansions for $\mathcal{D}_{k}(n)$ and $\mathcal{D}_{k}^{0}(n)$.

12. Prove that $\eta_{4}(n) \equiv 0(\bmod 5)$ if $n \equiv 24(\bmod 25)$.

13. Develop a full combinatorial theory of congruences for the $N F_{k}(m, Q, n)$ analogous to the achievements for the ordinary rank and crank chronicled by Mahlburg [23] and Bringman-Ono [13]. 
14. F. Garvan [18] successfully utilized (2.4) in a very different generalization of Dyson's rank. Are there other "ranks" that may lie hidden in specializations of (2.4).

\section{References}

[1] A. K. Agarwal and G. E. Andrews, Rogers-Ramanujan identities for partitions with "N copies of N", J. Comb. Th., Ser. A, 45 (1987), 4049.

[2] S. Ahlgren and K. Ono, Addition and counting: The arithmetic of partitions, Notices of the Amer. Math. Soc., 48 (2001), 978-984.

[3] K. Alladi, The method of weighted words and applications to partitions, from Number Theory, S. David ed., Cambridge University Press, Cambridge, 1995, pp. 1-36.

[4] K. Alladi, G. E. Andrews and A. Berkovich, A new four parameter qseries identity and its partition implications, Invent. Math., 153 (2003), $231-260$.

[5] G. E. Andrews, Problems and prospects for basic hypergeometric functions, fr. Theory and Appl. of Special Functions, R. Askey ed., Academic Press, New York, 1975, pp. 191-224.

[6] G. E. Andrews, The Theory of Partitions, Encycl. of Math. and Its Appl., G.-C. Rota ed., Addison-Wesley, Reading, 1976. (Reissued: Cambridge University Press, New York, 1998).

[7] G. E. Andrews, D. Crippa and K. Simon, $q$-Series arising from the study of random graphs, S.I.A.M. J. Discrete Math., 10 (1997), 41-56.

[8] G. E. Andrews and P. Freitas, Extension of Abel's lemma with $q$-series implications, Ramanujan Journal, 10 (2005), 137-152.

[9] G. E. Andrews and F. Garvan, Dyson's crank of a partition, Bull. Amer. Math. Soc., 18 (1988), 167-171.

[10] A. O. L. Atkin and F. Garvan, Relations between the ranks and cranks of partitions, Ramanujan J., 7 (2003), 343-366. 
[11] A. O. L. Atkin and H. P. F. Swinnerton-Dyer, Some properties of partitions, Proc. London Math. Soc., Ser. 3, 4 (1954), 84-106.

[12] K. Bringman and K. Ono, The $f(q)$ mock theta function conjecture and partition ranks, Invent. Math., 165 (2006), 243-266.

[13] K. Bringman and K. Ono, Dyson's ranks and Maass forms, (submitted).

[14] K. Dilcher, Some $q$-series identities related to divisor functions, Discrete Math., 145 (1995), 98-110.

[15] F. Dyson, Some guesses in the theory of partitions, Eureka, 8 (1944), 10-15 (Reprinted: Selected Papers, Amer. Math. Soc., Providence, 1996, pp. $51-56$.

[16] N. J. Fine, Basic Hypergeometric Series and Applications, Amer. Math. Soc., Providence, 1988.

[17] F. G. Garvan, New combinatorial interpretations of Ramanujan's partition congruences, Trans. Amer. Math. Soc., 305 (1988), 47-77.

[18] F. G. Garvan, Generalizations of Dyson's rank and non-RogersRamanujan partitions, Manuscr, Math., 84 (1994), 343-359.

[19] G. Gasper and M. Rahman, Basic Hypergeometric Series, Cambridge University Press, Cambridge, 1990.

[20] G. H. Hardy and E. M. Wright, An Introduction to the Theory of Numbers, 5th ed., Clarendon Press, Oxford, 1979.

[21] J. C. Kluyver, Vraagstuk XXXVII. (Solution by S. C. van Veen), Wiskundige Opgaven (1919), 92-93.

[22] P. A. MacMahon, The theory of modular partitions, Proc. Camb. Phil. Soc., 21 (1923), 197-204 (Reprinted: Coll. Papers, Vol. 1, M. I. T. Press, Cambridge, 1978, pp. 1090-1097.

[23] K. Mahlburg, Partition congruences and the Andrews-Garvan-Dyson crank, Proc. Nat. Acad. Sci, 102 (2005), 15373-15376.

[24] K. Ono, Distribution of the partition function modulo $m$, Annals of Math., 151 (2000), 293-307. 
[25] K. Ono, Arithmetic of the partition function, Proc. NATO Adv. Study Inst. on Special Functions, Special Functions 2000, eds. J. Bustoz and S. Suslov, Kluwer, New York, 243-253.

[26] S. Ramanujan, The Lost Notebook and Other Unpublished Papers, Intro. by G. E. Andrews, Narosa, New Delhi, 1997.

[27] J. J. Sylvester, Note on the paper of Mr. Durfee, Johns Hopkins Univ. Circulars 2 (1883), pp. 23, 24; 42, 43. (Reprinted: Coll. Papers, Vol. 3, pp. 660-662, Chelsea, New York, 1973).

[28] J. J. Sylvester, A constructive theory of partitions ..., Amer. J. Math., 5 (1882) 251-330, 6 (1884), 334-336. (Reprinted: Coll. Papers, Vol. 4, pp. 1-83, Chelsea, New York, 1973).

[29] K. Uchimura, An identity for the divisor generating function arising from sorting theory, J. Comb. Th., Ser. A, 31 (1981), 131-135.

[30] G. N. Watson, The final problem, J. London Math. Soc., 11 (1936), 55-80.

THE PENNSYLVANIA STATE UNIVERSITY

UNIVERSITY PARK, PA 16802 USA

Email: andrewsmath.psu.edu 